\title{
Stellar and Molecular Gas Kinematics of NGC1097: Inflow Driven by a Nuclear Spiral ${ }^{1}$
}

\author{
R.I. Davies \\ Max Planck Institut für extraterrestrische Physik, Postfach 1312, 85741, Garching, Germany \\ davies@mpe.mpg.de \\ and \\ W. Maciejewski \\ Astrophysics Research Institute, Liverpool John Moores University, Twelve Quays House, Egerton Wharf, \\ Birkenhead CH41 1LD, UK \\ and \\ E.K.S. Hicks, L.J. Tacconi, R. Genzel, H. Engel \\ Max Planck Institut für extraterrestrische Physik, Postfach 1312, 85741, Garching, Germany
}

\begin{abstract}
We present spatially resolved distributions and kinematics of the stars and molecular gas in the central $320 \mathrm{pc}$ of NGC1097. The stellar continuum confirms the previously reported 3-arm spiral pattern extending into the central $100 \mathrm{pc}$. The stellar kinematics and the gas distribution imply this is a shadowing effect due to extinction by gas and dust in the molecular spiral arms. The molecular gas kinematics show a strong residual (i.e. non-circular) velocity, which is manifested as a 2-arm kinematic spiral. Linear models indicate that this is the line-of-sight velocity pattern expected for a density wave in gas that generates a 3-arm spiral morphology. We estimate the inflow rate along the arms. Using hydrodynamical models of nuclear spirals, we show that when deriving the accretion rate into the central region, outflow in the disk plane between the arms has to be taken into account. For NGC 1097, despite the inflow rate along the arms being $\sim 1.2 \mathrm{M}_{\odot} \mathrm{yr}^{-1}$, the net gas accretion rate to the central few tens of parsecs is much smaller. The numerical models indicate that the inflow rate could be as little as $\sim 0.06 \mathrm{M}_{\odot} \mathrm{yr}^{-1}$. This is sufficient to generate recurring starbursts, similar in scale to that observed, every $20-150 \mathrm{Myr}$. The nuclear spiral represents a mechanism that can feed gas into the central parsecs of the galaxy, with the gas flow sustainable for timescales of a Gigayear.
\end{abstract}

Subject headings: galaxies: active — galaxies: individual (NGC1097) — galaxies: kinematics and dynamics - galaxies: nuclei — galaxies: spiral — infrared: galaxies

\footnotetext{
${ }^{1}$ Based on observations at the ESO Very Large Telescope (076.B-0098)
} 


\section{Introduction}

During the past decades there has been significant and increasing interest in mechanisms that can bring gas from remote locations in a galaxy to the nucleus, where it could fuel the central black hole. Such mechanisms would have to extract nearly all of the angular momentum from the gas if it is to be brought from a distance of a few kiloparsec to a few Schwarzschild radii from the central black hole. No unique mechanism has been found to operate over this entire range of radii, and the evidence is growing that no such single mechanism exists (Shlosman et al. 1990; Mulchaey \& Regan 1997; Martini et al. 2003; Hunt \& Malkan 2004; Wada 2004). In particular, it has been found that active star formation takes place in the innermost few parsecs of galaxies (Fabian et al. 1998; Levenson et al. 2001; Wada \& Norman 2002; Cid Fernandes et al. 2004; Thompson et al. 2005; Davies et al. 2007; Ballantyne 2008), which would disturb any flow pattern established at larger scales.

On scales of kiloparsecs, the most efficient drivers of gas inflow involve gravity torques, especially coming from passing companions, and non-axisymmetries in the disk, like bars (Combes 2003; Kormendy \& Kennicutt 2004; Garciá-Burillo et al. 2005). Observations show that the presence of companions correlates with star-forming activity in centres of galaxies but not with activity related to the central black hole (Schmitt 2001; Li et al. 2008a,b). A similar situation holds for bars, with Seyfert galaxies showing at best only a marginal statistical excess of bars with respect to non-Seyfert galaxies (Ho et al. 1997; Mulchaey \& Regan 1997; Laine et al. 2002; Combes 2003). Shlosman et al. (2000) even reported a slight deficiency of strong bars in Seyfert galaxies. Gas inflowing along the bar often settles on star-forming nuclear ring, about $1 \mathrm{kpc}$ from the galaxy centre, and inflow inside the ring is small (Piner et al. 1995; Regan \& Teuben 2003). The inflow may be extended inwards in nested bars, as proposed by Shlosman et al. (1989), but dynamical constraints on nested bars may prohibit inflow (Maciejewski et al. 2002). Englmaier \& Shlosman (2000) noticed that inside of strong inflow in a bar, instead of a nuclear ring, a nuclear spiral may form. Inflow in the bar along straight shocks curves towards the centre and turns into a spiral pattern. In the models of Englmaier \& Shlosman (2000) this gaseous nuclear spiral is no longer a shock, but a wave well described by the linear density wave theory. To the contrary, hydrodynamical models by Maciejewski (2004b) showed that the nuclear spiral can propagate to the centre of a galaxy as a shock in gas. Strong streaming motions (i.e. large velocity residuals), which take the form of kinematic spiral arms, are expected in the nuclear spiral shock (Maciejewski 2006). Kinematic spiral arms were already observed in emission from ionized gas in NGC 1097 (Fathi et al. 2006), NGC 6951 (Storchi-Bergmann et al. 2007) and M 83 (Fathi et al. 2008).

In this paper, we examine this mechanism with reference to NGC 1097, a nearby SBbc galaxy ( $\mathrm{D}=18 \mathrm{Mpc}$ for $H_{0}=70 \mathrm{~km} \mathrm{~s}^{-1} \mathrm{Mpc}^{-1} ; 1^{\prime \prime} \sim 85 \mathrm{pc}$ ). It has a strong large-scale bar and a $800 \mathrm{pc}$ radius circumnuclear ring that is rich in molecular gas (Gerin et al. 1988; Kohno et al. 2003; Hsieh et al. 2008) and vigorously forming stars (Hummel et al. 1987; Kotilainen et al. 2000). Inside this ring, a nuclear spiral extends down to the central few tens of parsecs (Prieto et al. 2005; Fathi et al. 2006). NGC 1097 hosts a weak AGN, classified as both a LINER and, from evidence of the broad line region (Storchi-Bergmann et al. 1993) and the hard X-ray excess (Iyomoto et al. 1996), as a type 1 Seyfert. Over the last decade or two, the AGN luminosity has gradually decreased (Storchi-Bergmann et al. 2003), and hydrogen recombination emission from the nuclear region is currently very weak (Davies et al. 2005, 2007). This is fortuitous, since it enables one to look very close in to the nucleus without being blinded by the AGN itself. We present SINFONI data for NGC 1097 extending out to a radius of 160 pc (see also Davies et al. 2007; Hicks et al. 2009), and analyse the distribution and kinematics of the stellar continuum and molecular gas emission in the context of the nuclear spiral.

In Section 2 we summarise the observations and data reduction, including the method used to extract 
kinematics and morphologies from the spectral features, an estimation of the point spread function (PSF), and the adopted orientation of the galaxy. We then analyse the stellar continuum in Section 3 and the molecular gas in Section 4, including an estimation of the gas surface density in and between the spiral arms. In Section 5 we discuss the dynamics of nuclear spirals, and then in Section 6 estimate the inflow rate for NGC 1097. We discuss the implications of our results in Section 7 before finally presenting our conclusions.

\section{Observations and Data Processing}

The data on which the present analysis of inflow is performed, have been presented previously in Davies et al. (2007) and Hicks et al. (2009). These papers focussed on the global properties of the stars and gas in samples of nearby active galaxies. Davies et al. (2007) (Section A2.4) showed that there was a kinematically (i.e. lower dispersion) and photometrically (i.e. excess stellar continuum) distinct stellar population in the nucleus, which had the properties of a short-lived and still young (i.e. <10 Myr old) starburst; and that the stars and molecular gas must be mixed. Hicks et al. (2009) (Section A.1) analysed the molecular gas, showing that it exhibits the characteristics expected for the large scale properties of the obscuring molecular torus. Specifically, the increase in the gas dispersion at radii less than $30 \mathrm{pc}$ is due to a thickening of the gas disk; and using also millimetre $\mathrm{CO}$ measurements, that the gas column density in this region is of order $10^{23} \mathrm{~cm}^{-2}$.

\subsection{Observations \& Reduction}

The observations of NGC 1097 were performed on the night of 10-Oct-2005 at the VLT with SINFONI, an adaptive optics near infrared integral field spectrograph (Eisenhauer et al. 2003; Bonnet et al. 2004). Data were taken with the $\mathrm{H}+\mathrm{K}$ grating (covering both bands simultaneously at a resolution of $R \sim 1500$ ), and with a pixel scale of $0.05^{\prime \prime} \times 0.1^{\prime \prime}$. Individual exposure times were $2 \times 300 \mathrm{sec}$, and 6 pairs of frames were taken in the sequence O-S-O-O-S-O, to facilitate background subtraction, yielding a total on-source integration time of 40 mins. The data were processed using the dedicated spred software package (Abuter et al. 2006), which provides similar processing to that for long slit data but with the added ability to reconstruct the datacube. The data processing steps were as follows. The object frames were pre-processed by subtracting sky frames, flat-fielding, and correcting bad pixels (which are identified from dark frames and the flatfield). The wavemap was generated, and edges and curvature of the slitlets were traced, all from the arclamp frame. The arclamp frame was then reconstructed into a cube, which was checked to ensure that the calibration is good. The pre-processed object frames were then also reconstructed into cubes. At this stage the wavelength calibration was fine-tuned for each cube separately with reference to the $\mathrm{OH}$ lines using a custom script. And in order to optimize the subtraction of the $\mathrm{OH}$ lines, the cubes were further processed using the method described in Davies (2007a). Following this, the cubes were spatially aligned using the bright nucleus as a reference, and finally combined. We note that during this process, an unusual but minor affect was imprinted into the data. This consists of slight changes between adjacent slitlets, which manifests themselves as small linear features in alternate rows, both in the flux and kinematics maps. Because the effect is small, and occurs at a spatial frequency significantly higher than the resolution, it has no impact on the analysis. The pixel scale of the final cube was $0.05^{\prime \prime} \times 0.05^{\prime \prime}$. Estimation of the spatial resolution (see below) was performed following extraction of the stellar continuum properties.

The standard star frames were similarly reconstructed into cubes. Telluric correction and flux calibration 
were performed with reference to the B6V star HD 28107. Because it is not easily possible to correct for all the absorption features in the H-band spectrum of such stars, the stellar spectrum was used only to derive the instrumental transmission as a function of wavelength. This was then combined with a theoretical model of the atmospheric transmission, which was adjusted to provide the best correction for the data. The zero-point derived from the standard star was consistent with that expected based on other standard stars taken at different times. The flux calibration was nevertheless cross-checked in $3^{\prime \prime}$ apertures using 2MASS data, and in smaller 1-3" apertures using broad-band imaging from NACO. The calibration of our data was consistent with the external data to $20-30 \%$.

\subsection{Emission/Absorption Line Characterisation}

The 2D distribution and kinematics of emission lines was found using the code LINEFIT developed by our group specifically for SINFONI applications (Davies et al. in prep.; see also Förster Schreiber et al. 2009). LINEFIT fits a function to the continuum-subtracted spectral profile at each spatial position in the datacube. The function is a convolution of a Gaussian with a spectrally unresolved template profile - an OH sky emission line. A minimisation is performed in which the parameters of the Gaussian are adjusted until the convolved profile best matches the data. During the minimisation, pixels in the data that consistently deviate strongly from the data are rejected. The uncertainties are boot-strapped using Monte Carlo techniques. Before extraction of the emission line properties, a $3 \times 3$ pixel median filter was used to smooth each spatial plane of the cube. While this affects the spatial resolution, it has no impact on the spectral properties. The key features of LINEFIT include: (i) the spectral resolution is implicitly taken into account by convolving the assumed emission line profile with a template line (tracing the effective instrumental resolution) before performing the fits; (ii) weighted fits are performed according to three possible schemes - in this instance we used uniform (i.e. no) weighting, since the noise was set to be the rms of the fit to the continuum at each spaxel; (iii) formal uncertainties are computed from 100 Monte Carlo simulations, where the points of the input spectrum at each spatial pixel are perturbed assuming Gaussian noise properties characterized by the rms from the noise cube.

The 2D properties of the stellar absorption features are extracted using an almost equivalent code. The only differences are that the convolution is performed using spectra of template stars observed independently but in the same instrument configuration (pixel scale and grating); and the uncertainties are derived assuming that the extracted profile is well represented by a Gaussian (an assumption that is not made in LINEFIT). As for the emission line, each spatial plane was smoothed with a $3 \times 3$ pixel median filter before performing the extraction.

During this procedure, the stellar and non-stellar continua are separated using the method described in Section 3.1 of Davies et al. (2007). Basically, stellar population synthesis models, as well as observations of galaxies, show that the equivalent width of the $2.3 \mu \mathrm{m}$ CO bandhead integrated over an evolving stellar cluster is approximately independent of age (for ages greater than $10 \mathrm{Myr}$ ). Any significant downward deviations from this value can be attributed to dilution by non-stellar continuum. At our resolution, within the central $0.5^{\prime \prime}$ of NGC 1097, non-stellar emission comprises slightly over $50 \%$ of the $2.3 \mu \mathrm{m}$ continuum. Within $1^{\prime \prime}$ and $2^{\prime \prime}$ apertures, this fraction drops to $40 \%$ and $35 \%$ respectively. 


\subsection{PSF Estimation}

There are a multitude of ways to derive the PSF from adaptive optics data, five of which are described in Davies (2007b). With AGN, it is usually possible to estimate the PSF from the science data itself, removing any uncertainty about spatial and temporal variations of the PSF due to atmospheric effects. For NGC 1097, the resolution was determined from the non-stellar K-band continuum. This will be unresolved in all but the nearest AGN since at near infrared wavelengths it is expected to originate from a region no more than 1-2 pc across. The non-stellar continuum is one of the frames output during the extraction of the stellar properties, and therefore already includes the effects of the spatial smoothing that was applied to the cube.

We have fit an analytical function to the PSF. Since the Strehl ratio achieved is relatively low, even a Gaussian is a reasonable approximation. However, we have used a Moffat function, which achieves a better fit because it also matches the rather broad wings that are a characteristic of partial adaptive optics correction. If one applies the concept of 'core plus halo' to this PSF, then the Gaussian fit would represent just the core while the Moffat fits the entire 'core plus halo'. Both functions yield a K-band FWHM of 0.25 ". Integrating both of these functions indicates that about $75 \%$ of the flux is within the 'core', and it is thus this component which dominates the PSF.

\subsection{Position Angle and Axis Ratio}

The position angle and axis ratio for the central few hundred parsecs of NGC 1097 have been derived previously for these SINFONI data. For each of these parameters, Davies et al. (2007) and Hicks et al. (2009) determined very similar values (i.e. to within a few degrees) from both the stellar velocity field and the gas velocity field. These values also agree well with those estimated by Storchi-Bergmann et al. (2003) and Fathi et al. (2006). We have adopted typical values here: a position angle of $-49^{\circ}$ and an axis ratio of 0.85 (i.e. inclination of $32^{\circ}$ ).

\section{Stellar Distribution and Kinematics}

Prieto et al. (2005) showed that although the total J-band continuum increased smoothly towards the nucleus, the residuals - after subtracting elliptical isophotes - revealed a 3-arm spiral structure in absorption. This 3-arm structure is confirmed by our SINFONI data. In Fig. 1 we show the K-band continuum distribution, together with the residual after fitting and subtracting elliptical isophotes. Prieto et al. (2005) decomposed the near infrared continuum from the central few arcsec into a contributions from a bulge and a point source. Davies et al. (2007) confirm the presence of a bulge, measuring $V / \sigma<0.8$ out

to $2^{\prime \prime}$. But in addition, both they and Emsellem et al. (2001) found evidence for a drop in dispersion close to the nucleus, indicating a kinematically distinct stellar population within the central arcsec. While our primary interest is in the residuals rather than the fit itself, our fitting procedure needs to be sufficiently representative. As a result, in order to allow for multiple stellar components as well as possible isophotal twists of the bulge, we have left the axis ratio and the position angle, as well as the center, as free parameters for each isophote. These extra degrees of freedom, by making it possible to remove global gradients that would otherwise have remained, yield a more uniform residual with clearly defined structures. There are three arms in the residual: the northeastern and the southwestern arms are strong, and the third arm, towards the northwest, is weaker. The overlay shows that they do trace the same pattern, although less distinct, as in the J-band NACO data presented by Prieto et al. (2005). 
The SINFONI data have the advantage of probing also the kinematics, and we present the stellar velocity field - together with its residual - in Fig. 2. Again, our primary aim is to quantify the residual structures; and the reader is recommended to look at Section A2.4 of Davies et al. (2007) as well as Section A.1 of Hicks et al. (2009) for details about the bulk properties of the gas and stellar systems themselves. The residual was created by fitting and subtracting a velocity map for a simple axisymmetric disk model generated by the code DYSMAL, which is described in Cresci et al. (2009). The code produces a data cube with 2 spatial and 1 velocity axis, from which a velocity field can be extracted in the same way as for real data. The input parameters needed include the mass distribution and geometrical configuration of the disk. In this case, the orientation (position angle and inclination) of the disk were fixed as in Section 2.4; the systemic velocity, as well as the total mass and its distribution (described by a Sersic function with the defining parameters Sersic index $n$ and effective radius $r_{e}$ ) were allowed to vary. These 4 parameters provide a convenient way to generate a velocity field appropriate for a disk. Their values, which are in themselves unimportant, were adjusted by comparing the output velocity field to that extracted from the data and minimising the difference, taking into account the noise in each spaxel. We emphasize that because we have not included the dispersion in the minimisation and mass estimation, the mass derived does not necessarily represent the true dynamical mass in the central region of NGC 1097. However, the purpose of this procedure was solely to derive a best-fitting velocity field, and for that it is sufficient.

The residual of the stellar velocity field shows no coherent structures above the noise level. This lack of evidence for non-circular motions implies that the stars themselves are not inherently associated with the spiral structure. This is perhaps not surprising given the rather high velocity dispersion of $150 \mathrm{~km} \mathrm{~s}^{-1}$ at radii from $0.5^{\prime \prime}$ to the edge of the SINFONI field (Davies et al. 2007). This implies that the kinematics are dominated by random motions rather than ordered rotation, hence we are in fact seeing the bulge stars. In this case, the 3-armed spiral structure seen in the stellar continuum must be a passive effect, most likely caused by absorption from the true gaseous arms. This was a hypothesis already put forward by Prieto et al. (2005), and one which we shall examine in more detail in Section 4.

\section{Molecular Gas}

\subsection{Gas Distribution and Flux Residuals}

Within the spectral range for which SINFONI data were obtained, warm molecular gas at temperatures of $1000-3000 \mathrm{~K}$ can be traced by the $2.12 \mu \mathrm{m} 1-0 \mathrm{~S}(1)$ emission line which arises from $\mathrm{H}_{2}$. The $2 \mathrm{D}$ distribution of this line was extracted as described in Section 2.2, and is presented in Fig. 3. As for the continuum, we have fitted and subtracted elliptical isophotes to obtain the residual flux distribution. This time, because the gas is expected to reside in a thin planar disk, we fixed a common center, position angle, and axis ratio during the fitting process.

Like the stellar residual flux, presented above, the molecular gas residuals also reveal an asymmetric distribution of spiral morphology. Three arms are clearly present, with the northwest one somewhat weaker and more diffuse. What is immediately apparent is the close spatial coincidence between the positive $\mathrm{H}_{2}$ residuals and the negative stellar residuals. This correlation of 1-0 S(1) $\mathrm{H}_{2}$ emission with stellar continuum absorption lends strong support to the hypothesis that the structures in stellar continuum are the result of obscuration.

A closer inspection indicates some spatial offset between the stellar continuum and molecular flux residuals: the brightest 1-0 S(1) emission tends to lie along the inside edge of the arm traced by the J-band 
stellar residuals. This offset is clearly seen in the northeastern arm close to the nucleus (Fig. 3), and along the southwestern arm. It is perhaps to be expected, since the extinction is roughly proportional to the total amount of gas, while the 1-0 S(1) emission traces only gas that is heated, probably by shocks, as it enters the arm along its inside edge (see Section 5). Therefore we refer to the spiral arms traced by the J-band stellar residual as the 'morphological' arms, since we believe they better represent the true location of gas than the 1-0 S(1) emission line. The picture presented above is complicated about $1^{\prime \prime}$ to the southwest of the nucleus. There the northwestern and southwestern arms appear to meet, which may be responsible for the shift in the brightest 1-0 S(1) emission.

Remarkably, there is an additional hint of the 3-armed spiral structure in the $\mathrm{HCN}(1-0)$ data presented by Kohno et al. (2003) that was obtained with the Nobeyama Millimeter Array. Although the resolution was only $4^{\prime \prime} \times 10^{\prime \prime}$, the central peak clearly has an extended triangular form, in contrast to the smoothly elliptical beam. The vertices of this triangle point in the directions of the morphological arms on scales of a few hundred parsecs. An equivalent structure is seen at best only weakly in $\mathrm{CO}(1-0)$ data (Kohno et al. 2003; Hsieh et al. 2008), suggesting that the arms are composed preferentially of denser $n_{H}>10^{4} \mathrm{~cm}^{-3}$ gas.

It is clear from the $\mathrm{H}_{2}$ residual flux map in the central panel of Fig. 3 that as one moves inwards along the arms, the intensity of the residual 1-0 S(1) flux increases. However, this effect disappears if the residual flux is compared to the total line flux in each spaxel, as shown by the ratio map in the far right panel. This suggests that the flux increase in the residuals is related to the global flux increase at smaller radii.

One possible reason for this could be an increase in the fraction of warm to cold $\mathrm{H}_{2}$, perhaps due to more efficient excitation at small radii, for example by the nuclear starburst reported by Davies et al. (2007) and Storchi-Bergmann et al. (2005). An alternative could simply be an increase in the volume filling factor of molecular clouds at small radii, an effect that would be expected given the trend for greater mass surface density at smaller radii shown in Fig. 7 of Davies et al. (2007).

The molecular spiral structure can be traced to within about $0.2^{\prime \prime}$ of the centre, a radius that is formally spatially resolved (the HWHM from Section 2.3 is $0.1^{\prime \prime}$ ). At this point the residual $\mathrm{H}_{2}$ (in both the flux and ratio maps of Fig. 3) decreases noticeably. There could be two reasons for this. The width of the arms is about $0.4^{\prime \prime}$. If this size scale is maintained into the centre, one would no longer expect to discern separate arms closer in than a radius of about $0.2^{\prime \prime}$. As a result, any flux closer in than this would therefore be accounted for during the isophotal fitting and not show up in the residual map. Alternatively, it could be associated with the increase in the gas velocity dispersion at about this radius (Davies et al. 2007; Hicks et al. 2009), suggesting that the gas kinematics are more dominated by random motions, and hence disk dynamical phenomena are no longer supported.

\subsection{Extinction and gas densities}

As we noted above, the spiral structure is present in stellar continuum images because of a difference in extinction between the arms and the interarm region. The extinction itself can be derived by comparing the spectral slope of the K-band continuum in our SINFONI data to that of stellar templates, as described in Hicks et al. (2009). We have used K-type templates, although there is little difference in the intrinsic slope for any reasonable stellar population. The low gas velocity dispersion at radii outside $25 \mathrm{pc}$ (i.e. the region of interest here) reported by Davies et al. (2007) and Hicks et al. (2009) indicates that the gas must lie in a disk, hence the dust associated with it obscures only half of the stellar bulge light and a screen approximation is the appropriate model to use for this extinction. The resulting average extinction at radii 
$0.3-2.0^{\prime \prime}$ is $A_{V}=4.9 \pm 1.9$. If a constant dust to gas ratio is assumed, one can obtain the gas column density in the disk, which is directly proportional to $A_{V}$.

Interestingly, one can use the contrast between the arm and inter-arm regions to relate the interarm extinction to the extinction in the arm, which gives the arm/interarm gas density ratio if the dust fraction in the gas is constant. If we denote the flux intensity on an arm as $F_{a}$, the inter-arm flux intensity as $F_{i}$, and the flux from the isophotal fit as $F_{f}$, then one can define a contrast ratio $C$ as the ratio of the difference between the arm and inter-arm regions to the total flux (as represented by the fit)

$$
C=\left(F_{i}-F_{a}\right) / F_{f}
$$

As argued above, for dust distributed in the plane of the galaxy, the appropriate extinction model is that for a screen obscuring only half the light. Thus, denoting the intrinsic flux intensity from the bulge as $F$, we can write $F_{a}=0.5 F\left(1+x_{a}\right)$ and $F_{i}=0.5 F\left(1+x_{i}\right)$ where $x_{a}$ and $x_{i}$ are the respective arm and inter-arm factors by which the obscured half of the bulge is attenuated, hence the extinction $A$ is given by

$$
A=-2.5 \log x
$$

Since at any point the fitted flux $F_{f}$ is approximately the same as the interarm flux $F_{i}$, we find that $\left(x_{i}-x_{a}\right) /\left(1+x_{i}\right)=C$, and hence that there is a linear relation between $x_{a}$ and $x_{i}$ :

$$
x_{a}=(1-C) x_{i}-C
$$

This equation, combined with (2), indicates how the extinction in the arm depends on the interarm extinction for an observed contrast ratio $C$. The far right panel in Fig. 1 indicates that the typical value of $C$ over the central few arcsec in the K-band is $0.10-0.15$, i.e. that the K-band continuum is about $10-15 \%$ fainter on the arms. For $C$ values in this range, the arm/interarm extinctions should lie in between the two thick red lines in Fig. 4.

The contrast ratio observed in our SINFONI K-band data is essentially the same as in the NACO Kband image presented by Prieto et al. (2005). In their J-band data, the typical value of $C$ over the central few arcsec is $0.15-0.20$. Since $A_{V}=3.6 A_{J}=10 A_{K}$, one gets a second set of equivalent lines for J-band in Fig. 4. The contrast ratio in the J-band indicates that the arm/interarm extinctions should lie in between the two thick blue lines. Thus in principle, having the contrast between the arm and interarm regions in more than one band should enable one to independently determine the extinction and the extinction ratio. In our case, the combined observed ranges of the contrast ratio in J-band and K-band confine the possible extinction and arm/interarm extinction ratio to a narrow range of values. In particular, these two constraints alone are sufficient to determine the extinction, without having to know a priori the intrinsic colours.

If we take the interarm extinction as an approximation for the average extinction, its value is in excellent agreement with $A_{V}=4.9 \pm 1.9$ obtained above by independent means. As shown in Fig. 4, combining the constraints coming from the slope of the spectrum with those of the contrast ratios, yields a tight constraint on the extinction and arm-interarm extinction ratio. Our adopted values are therefore $A_{V}=4 \pm 1$, and an extinction ratio of $2.0 \pm 0.3$. Thus the extinction along the arms is $A_{V}=8.0 \pm 2.3$.

We note that the extinction we find is higher than the $A_{V} \sim 1$ derived by Prieto et al. (2005). The reason is two-fold: these authors assumed that the intrinsic $J-H$ and $H-K_{s}$ colours of the bulge were those measured at a radius of $2.5^{\prime \prime}$; and they assumed that all the light from the bulge was extincted. Correcting for these effects will increase their extinction significantly, making it more consistent with the value we have derived. 
If the dust fraction in the gas is constant, the arm-interarm extinction ratio is equal to the gas density ratio, and does not depend on the actual dust fraction. Thus we estimate the arm-interarm gas density ratio in the spiral to be $2.0 \pm 0.3$. On the other hand, in order to estimate the actual gas column density in the disk, one needs to rely on the relation between extinction and total hydrogen column density along the line of sight, $n_{H}^{s k y}$, which for a standard gas-to-dust ratio of 100 is $n_{H}^{s k y}\left(\mathrm{~cm}^{-2}\right)=1.9 \times 10^{21} A_{V}$ (Tokunaga 2000). Thus the total projected hydrogen column density between the arms is $n_{H}^{s k y}=(7.6 \pm 1.9) \times 10^{21} \mathrm{~cm}^{-2}$. The corresponding total deprojected gas surface density in the galaxy disk, $\Sigma_{\text {gas }}$, is expressed by

$$
\Sigma_{\text {gas }}\left[\mathrm{M}_{\odot} \mathrm{pc}^{-2}\right]=\Sigma_{\text {gas }}^{\text {sky }} \cos i=1.36 \Sigma_{H}^{\text {sky }} \cos i=10.9 \times 10^{-21} \cos i n_{H}^{s k y}\left[\mathrm{~cm}^{-2}\right],
$$

where $i$ is the inclination of the obscuring disc, and the 1.36 factor is the helium correction applied to the total hydrogen density $\Sigma_{H}$. In between the arms, we get $\Sigma_{\text {gas }}=70 \pm 17 \mathrm{M}_{\odot} \mathrm{pc}^{-2}$. Along the arms, the corresponding values are $140 \pm 40 \mathrm{M}_{\odot} \mathrm{pc}^{-2}$. If we assume that the arms are as thick as they are wide, about $0.4^{\prime \prime}$, this implies a mean total hydrogen density in the arms of $100-150 \mathrm{~cm}^{-3}$. Since the gas is likely to exist in clouds rather than being uniformly distributed, it is consistent with the arms being detected in $\operatorname{HCN}(1-0)$ by Kohno et al. (2003), which traces gas denser than $\sim 10^{4} \mathrm{~cm}^{-3}$. These two results are consistent when volume filling factor is no more than $1-2 \%$.

Based on the $\mathrm{CO}(2-1)$ molecular line luminosity, and the (2-1) to (1-0) line ratio reported by Hsieh et al. (2008), we can estimate the average central molecular hydrogen column density within their observed beam of $3.1^{\prime \prime} \times 4.1^{\prime \prime}$. Using the conversion factor of $1.4 \times 10^{20} \mathrm{~cm}^{-2}\left(\mathrm{~K} \mathrm{~km} \mathrm{~s}^{-1}\right)^{-1}$ between the (1-0) line intensity and column density of molecular gas, as in Hicks et al. (2009), we estimate this density at $2.8 \times 10^{22} \mathrm{~cm}^{-2}$, corresponding to the total deprojected gas surface density in the galaxy disk of $260 \mathrm{M}_{\odot} \mathrm{pc}^{-2}$. Even if all the gas in this central region were molecular, this estimate is 3.7 times higher than the interarm surface density derived above from the stellar continuum extinction. We discuss the implications of this possible difference in mass surface density in Section 7.

\section{3. $\quad \mathbf{H}_{2}$ Kinematics and Velocity Residuals}

The 2D kinematics of the $\mathrm{H}_{2}$ have been extracted as described in Section 2.2 and are presented in Fig. 5. As for the stellar kinematics, we have fitted an axisymmetric disk model to the velocity field, for a fixed position angle and axis ratio, allowing the mass and its distribution to vary. Subtracting this model reveals a clear 2-armed velocity residual. This 2-arm kinematic spiral pattern winds in the same direction as the 3 -arm morphological pattern seen in the residual flux map, but more tightly. Understanding how the two patterns are related, and how they might relate to gas inflow or outflow is developed in Section 5.

Intriguingly, a spiral structure in the velocity residual was already reported by Fathi et al. (2006) based on their analysis of the [NII] emission line. However, Fathi et al. (2006) were unable to fully explain their data, probably because their interpretation was mislead by attempting to identify a 3 -arm spiral in the kinematics: they argued that there were two 'blue-shifted' arms in the residual and one 'red-shifted' arm. However, the locations of these features do not correspond to the morphological arms. We postulate that Fathi et al. (2006) missed one 'red-shifted arm', towards the north, in between their two 'blue-shifted' arms. Adding this arm puts their result in accordance with our 2-arm kinematic spiral: a 2-arm kinematic spiral has two velocity minima ('blue-shifted' arms) and two maxima ('red-shifted' arms). It is clear that between two minima a maximum should exist. Once this missing 'red-shifted' arm is added, the kinematic arms traced by Fathi et al. (2006) in ionised gas kinematic residuals correspond very well to what we trace in molecular gas kinematic residuals (Fig. 6). Two arms marking velocity minima in molecular gas continue as 
arms labeled 1 and 3 by Fathi et al. (2006). One arm marking velocity maximum that in our data heads towards south-west, continues as the arm labeled 2 by Fathi et al. (2006). Another velocity-maximum arm in our data heads towards the north, where it connects to a region of positive velocity residual in the ionized gas, which constitutes the arm missed by Fathi et al. (2006). Kinematic residuals are less consistent between ionized and molecular gas in a region about $1^{\prime \prime}$ to the east and slightly north of the nucleus, where we find a positive residual but Fathi et al. (2006) find a negative residual. The reason for this may be associated with the map of the [NII] velocity dispersion in their paper, which shows a region of very high dispersion at this location.

The amplitude of $\mathrm{H}_{2}$ velocity residuals $\left(40 \mathrm{~km} \mathrm{~s}^{-1}\right.$ before correcting for inclination) is similar to that measured by Fathi et al. (2006) for [NII] emission. Thus the intrinsic amplitude of radial motions observed in the central regions of NGC 1097, after deprojection, reaches $75 \mathrm{~km} \mathrm{~s}^{-1}$. Interestingly, it is apparent from Fig. 5, that the most consistent feature of the velocity field in NGC 1097 is gas outflow between the morphological arms, seen within 1 arcsec from the centre along the minor axis. In NGC 1097, the velocity dispersion measured for the warm $\mathrm{H}_{2}$ gas in the central $320 \mathrm{pc}$ is about $50 \mathrm{~km} \mathrm{~s}^{-1}$ (Davies et al. 2007; Hicks et al. 2009). Since bright 1-0 S(1) emission is expected for shock velocities of $20-40 \mathrm{~km} \mathrm{~s}^{-1}$ (the molecules dissociate at higher shock velocity) this measurement is likely to be an upper limit to the true dispersion (see Hicks et al. 2009). Applying a quadrature correction yields an intrinsic dispersion of $30-45 \mathrm{~km} \mathrm{~s}^{-1}$, which is consistent with the $20-40 \mathrm{~km} \mathrm{~s}^{-1}$ [NII] dispersion measured by Fathi et al. (2006). This value is also characteristic of the central velocity dispersion measured in other galaxies from CO emission (e.g. Schinnerer et al. 2006), which better represents the average state of the dominant gas phase.

\section{Dynamics of Nuclear Spiral Structures}

In the previous sections, we have described how our high spatial resolution observations of gas morphology and kinematics in the inner 320 pc of NGC 1097 recover a spiral pattern that extends to the innermost $20 \mathrm{pc}$ of the galaxy. The pattern is present both in morphology of the warm gas, traced by the $\mathrm{H}_{2}$ emission, and in the gas velocity residuals. Below we show that all observed characteristics of the spiral are consistent with a density wave propagating in a gaseous disc. In principle, such a density wave can either be self-amplifying, like in the standard Lin-Shu theory, or driven by a rotating non-axisymmetric perturbation in the stellar potential. In order for the wave in gas to be self-amplifying, it has to overcome the stabilizing effect of the stellar component, in which the spiral structure is apparently absent, and of the central supermassive black hole. Simple application of the Toomre stability criterion indicates that the central gaseous disc in NGC 1097 can become unstable only when gas surface densities are well above $1000 \mathrm{M}_{\odot} / \mathrm{pc}^{2}$ at $100 \mathrm{pc}$ from the galaxy centre, and still higher at smaller radii. This is significantly more than the value we have estimated in Section 4.2. Thus we imply that the nuclear spiral in NGC 1097 is a driven density wave, the driver being possibly one of the bars or another rotating non-axisymmetric perturbation in the total gravitational potential.

Density waves driven in gas by a rigidly rotating external potential have been extensively studied (e.g. Goldreich \& Tremaine 1979; Maciejewski 2004a,b). In Appendix A, we summarize the derivation of characteristics of the resulting spiral pattern within the linear theory. We note that the geometry and the amplitude of the spiral does not depend on the triggering mechanism (Eq. A8), and the amplitude of radial velocities is only a weak function of the number of arms (Eq. A12). The theory implies (Eq. A15) that the velocity residuals of an $m$-arm spiral density wave should be dominated by an $(m-1)$-arm kinematic spiral, a correspondence originally noticed by Canzian (1993). In NGC 1097, the photometric spiral seems to consist 
of three arms, as originally noticed by Prieto et al. (2005), but our observation of the kinematic spiral (Fig. 5) indicates that this has only two arms, in full agreement with the linear theory. Eq. (A16) shows that within the limits of the linear theory the arms of the kinematic spiral should be more tightly wound than those of the morphological spiral. As is apparent from Figs. 3 and 5, this is also observed in NGC 1097. Eqs. (A9) and (A10) indicate that the radial velocity is shifted in phase from the density perturbation by $180^{\circ}$ which means that the density maximum corresponds to the maximum in inflow velocity, while maximum radial outflow is expected at the density minimum (see also the central panel of Fig. 8). The most prominent kinematical feature that we observe in NGC 1097, outflow between the spiral arms, is in full agreement with this prediction.

Despite these successes of linear theory in describing what is observed, we need to be cautious because, as we show below, there are also some differences between the theory and observations. Instead, beyond the linear regime, properties of nuclear spirals associated with a shock wave propagating in gas can be analysed using the detailed hydrodynamical models from Maciejewski (2004b). Although these models were constructed for nuclear spiral shocks of 2-arm morphology and triggered by a bar, a limited quantitative comparison between the models and NGC 1097 is possible.

In Eq. (A12) we show in the linear approximation that the arm/interarm density amplitude $(1+\epsilon) /(1-\epsilon)$ corresponds to the amplitude of radial velocity $\epsilon c$, where $\epsilon$ is much smaller than 1 , and $c$ is the isothermal sound speed, or one-dimensional velocity dispersion in gas. The amplitude of radial motions observed in NGC 1097 is significantly larger than the velocity dispersion $\left(75 \mathrm{~km} \mathrm{~s}^{-1}\right.$ and $30-40 \mathrm{~km} \mathrm{~s}^{-1}$, respectively, see Section 4.3). This indicates that the spiral wave observed in NGC 1097 is beyond the linear density wave regime, and most likely constitutes a shock in gas. Eq. (A5) shows that in the linear regime, the tangent of pitch angle of the spiral wave is proportional to the $c / V_{\text {rot }}$ ratio, where $V_{\text {rot }}$ is the rotational velocity. For the central $320 \mathrm{pc}$ of NGC 1097 this ratio is about 0.25 , while tangent of the observed pitch angle $\left(\sim 60^{\circ}\right)$ is much larger, about 1.7. The nuclear spiral in NGC 1097 is too open to be well described by a linear theory, but both large pitch angle and large amplitude of radial velocity can be accounted for when one goes beyond the linear regime, to the regime when the spiral constitutes a shock in gas. As discussed above, we can do this by comparison to hydrodynamical models.

In the left panel of Fig. 7, we present the characteristics of the inner few hundred parsecs of model 8S20r from Maciejewski (2004b) that best represents a nuclear spiral associated with a shock. As already predicted by the linear theory, there is strong inflow in the morphological spiral arms in the model (red contours), and equally strong outflow between them (blue contours). Maciejewski (2004b) identified the strong gradient of radial velocity on the inside edge of the morphological spiral arm as a shock in the gas, using negative divergence of the full two-dimensional velocity field as the shock indicator. The reason for this flow pattern is that the gas inflowing in the arm preserves some angular momentum, hence it does not fall onto the galaxy centre, but passes it by at a certain distance, and continues as a diverging outflow, in which the gas density decreases. This now low-density, outflowing gas hits the inside edge of the other spiral arm. This collision maintains the presence of the shock on the inside edges of the arms. In the shock the gas is again compressed, and because of losing angular momentum it starts flowing inwards, so the cycle repeats. Thus the morphological spiral is always downstream from the spiral shock, which can explain why in NGC 1097 the photometric spiral in the stellar residual, coming from obscuration caused by dust associated with high density gas, is downstream from the spiral in 1-0 S(1) emission, which traces warm, recently shocked molecular gas.

The nuclear spiral in the model is loosely wound, which well represents the morphological spiral observed in NGC 1097. The amplitude of the radial velocity in the model reaches $60 \mathrm{~km} \mathrm{~s}^{-1}$, compared to $75 \mathrm{~km} \mathrm{~s}^{-1}$ 
observed in NGC 1097 (Section 4.3). The speed of sound in the isothermal gas (the one-dimensional velocity dispersion) in the model is $20 \mathrm{~km} \mathrm{~s}^{-1}$, while estimates of this value for the centre of NGC 1097 are between 30 and $40 \mathrm{~km} \mathrm{~s}^{-1}$. Thus the observed Mach number is slightly lower than that in the model, implying that the observed shock is somewhat weaker than in the model. Proper modeling of the nuclear spiral shock would require taking into account the gas velocity dispersion rising towards galaxy center, which has not yet been done, and is beyond the scope of this paper. The ratio of highest density in the arm to the lowest interarm density in the model can be as high as 10, but more representative for comparison with the observations is the ratio of average densities in the arms and between the arms, where arms in the model are defined as regions of inflow (negative radial velocity in Fig. 7). This ratio is about 2.4, slightly higher than the arm/interarm density ratio of 2.0 implied by observations (Section 4.2). This again indicates that the nuclear spiral observed in NGC 1097 hosts a slightly weaker shock than the one in the model.

The photometric and the kinematic spiral in the model, as seen in the plane of the sky, are shown in the central and right panels of Fig. 7. A one-arm kinematic spiral in velocity residuals corresponds to the two-arm photometric spiral. Thus the relation already found in linear approximation holds also beyond the linear regime, in a nuclear spiral shock. As we noted above, this relation is also observed in NGC 1097. From Fig. 7 one can also see that the pitch angle of the photometric spiral is larger than the pitch angle of the kinematic spiral, so this prediction of the linear approximation holds also beyond the linear regime. This relation of pitch angles is also observed in NGC 1097 (Section 4.3).

\section{Gas Transport Rates}

The rate of radial gas transport $\dot{M}$ can be most simply estimated from the gas density in the spiral arms, and the radial velocity as

$$
\dot{M}=m \Sigma v_{r} \frac{W}{\sin \alpha},
$$

where $m$ is the number of arms, $\Sigma$ is the mean density of gas in the arm, $v_{r}$ is the mean radial velocity in the arm, $W$ is the arm width and $\alpha$ is the pitch angle of the arm. This estimate is valid for rather loosely wound spirals, with $\alpha \geq 45^{\circ}$, which is the case of NGC 1097 and model 8S20r from Maciejewski (2004b). In NGC 1097, the mean gas column density in the arms (see Section 4.2) is $100-180 \mathrm{M}_{\odot} / \mathrm{pc}^{2}$. The intrinsic mean radial velocity in the arm, after deprojection, is $75 \mathrm{~km} \mathrm{~s}^{-1}$, and we estimate that the arms are about $0.4 \operatorname{arcsec}(34 \mathrm{pc})$ wide. The pitch angle of the arms is about $60^{\circ}$. For these values, Eq. 5 gives the radial inflow rate of $1.23 \pm 0.35 \mathrm{M}_{\odot} \mathrm{yr}^{-1}$, which is quite significant.

However, already the linear theory indicates that the inflow in the arms is accompanied by the outflow between the arms (see the middle panel of Fig. 8). Beyond the linear regime, hydrodynamical modelling described in Section 5 shows that at galactocentric radii, where the nuclear spiral shock is present, most of the volume is occupied by gas that has positive radial velocity component, i.e. is outflowing. This can be seen in the left panel of Fig. 7, where blue contours that mark positive radial velocities enclose the majority of the volume. This outflow partially balances the inflow along the arms. However, in the presence of the shock, the gas on average loses angular momentum because of its dissipative nature, so there is net inflow in the models. For the flow in model 8S20r from Maciejewski (2004b), presented in Fig. 7, this inflow is below $0.01 \mathrm{M}_{\odot} \mathrm{yr}^{-1}$ at the radius of $100 \mathrm{pc}$. On the other hand, the flow parameters in model 8S20r that enter Eq. 5 are $m=2, \Sigma=100 \mathrm{M}_{\odot} / \mathrm{pc}^{2}, v_{r}=50 \mathrm{~km} \mathrm{~s}^{-1}, W=14 \mathrm{pc}$, and $\alpha=45^{\circ}$. For these values, Eq. 5 gives a radial inflow rate of $0.2 \mathrm{M}_{\odot} \mathrm{yr}^{-1}$. The discrepancy between the actual inflow in the model and the one estimated from Eq. 5 comes obviously from the equation not taking into account the interarm outflow. 
For NGC 1097 we cannot derive the inflow directly from the observed data, and we would like to correct the inflow estimated from Eq. 5 for the effects of outflow. The best we can do is to use the ratio of the actual to the estimated inflow in model 8S20r as a correction factor, as there are no other models that better reflect the observed nuclear spiral shock. As stated in Section 5, the characteristics of the density wave do not depend strongly on its driver or the number of arms at least in the linear regime. Moreover, the kinematics of the nuclear spiral observed in NGC 1097 are very similar to those in model 8S20r. With the correction factor estimated from the model being about 20, the corrected inflow to the centre of NGC 1097 should be about $0.06 \mathrm{M}_{\odot} \mathrm{yr}^{-1}$. However, this value is uncertain, as gas flow in the observed 3-arm spiral and in the modelled 2-arm spiral can differ because of the factors involved in generating the 3 -arm spiral, whose origin remains unclear (see Section 7.4). It is certain, however, that because of the outflow between the arms, the net inflow in NGC 1097 is smaller than $1.2 \mathrm{M}_{\odot} \mathrm{yr}^{-1}$.

\section{Discussion}

\subsection{Gas Mass and Density}

In order to estimate the average central gas surface density in NGC 1097, $\Sigma_{\text {gas }}$, we assumed that the gas is distributed uniformly. On the other hand, the mean volume density we find of $100-150 \mathrm{~cm}^{-3}$ is rather less than the $10^{4} \mathrm{~cm}^{-3}$ needed for bright $\operatorname{HCN}(1-0)$ emission. This implies that the gas distribution, at least in the arms, is most likely to be clumpy and confined to discrete clouds with a volume filling factor of only a few percent. Such dense clouds must be small: for the inter-arm column density we have estimated, it implies cloud sizes of no more than $0.2 \mathrm{pc}$ across. This suggests that the expression for extinction by a clumpy medium (Calzetti et al. 1994) may be more appropriate. Independent of the actual clouds size, this expression relates the effective optical depth $\tau_{\text {eff }}$ (i.e. that measured) to the average number of clouds $N$ along the line of sight and the optical depth through each cloud $\tau_{c l}$ as:

$$
\tau_{e f f}=N\left(1-e^{-\tau_{c l}}\right) .
$$

For such a distribution, some lines of sight will be relatively little obscured, while others may be highly obscured. And if the medium is optically thick on average, one can hide a considerable column density of gas that has almost no impact on the observed extinction. In this regime one would find that $N \tau_{c l}>\tau_{\text {eff }}$. We have used Eq. 6 together with the measured extinction $A_{K}=0.4$ to estimate the impact of a clumpy medium, and verified the result statistically. For $N$ greater than a few (implying cloud sizes $<0.1 \mathrm{pc}$ ), this expression yields a quantitative result very similar to that obtained by assuming a uniform dust screen; and even in the extreme case of $N=1$, it implies we have underestimated the column density by only about $20 \%$. The reason is simply that in the K-band (i.e. the observational waveband) individual clouds are not optically thick, so one is in the regime where $N \tau_{c l} \sim \tau_{\text {eff }}$.

The clumpiness of the ISM in NGC 1097 may help to understand the different appearances of the VLT NACO J- and K-band images, the $814 \mathrm{~nm}$ HST ACS and $550 \mathrm{~nm}$ HST WFPC2 images (presented in Prieto et al. 2005 and Fathi et al. 2006). In the J- and K-band images, the circumnuclear region appears relatively smooth, although the dust lanes are prominent in the residual when elliptical isophotes are subtracted. On the other hand, in the $814 \mathrm{~nm}$ image, a dusty structure is visible even in the direct image, and appears more like a flocculent spiral. Then at $550 \mathrm{~nm}$, the direct image is again relatively smooth. In terms of the clumpy extinction model, the following argument is valid as long as there are a few clouds along the line of sight, and is not dependent on the exact number. For simplicity let us assume that there are on average $N=4$ along the line of sight, so that in the K-band each cloud is optically thin with $\tau_{c l, K}=0.1$. On the other hand 
at $550 \mathrm{~nm}$ even individual clouds will be optically thick with $\tau_{c l, V}=1$. Thus in the K-band, the extinction traces the global structure of the dust through the whole disk. As a result one sees light from the bulge stars from in front of the disk and, attenuated, from behind. In the V-band, nearly every line of sight is fully obscured by the disk (statistically only $2 \%$ of all lines of sight would not encounter a cloud). Thus one sees primarily light from bulge stars in front of the disk, and the dust structure appears only weakly. At $814 \mathrm{~nm}$, one is in an intermediate situation, and hence much more sensitive to the number of clouds in any particular line of sight. The structure in the dust distribution therefore shows up more strongly, and is also influenced by relatively small changes in extinction. As a result one sees details in the structure as well as the global 3-arm spiral. We note that the actual situation may differ from this simplified picture, since the gas surface density we have derived implies, via the Kennicutt-Schmidt relation (Kennicutt 1998), that there is a low level of on-going star formation in the disk. However, we believe our simplified analysis is sufficient to understand what is observed and derive meaningful quantities.

The value for $\Sigma_{\text {gas }}$ that we derived in Section 4.2 , oscillating between $140 \mathrm{M}_{\odot} \mathrm{pc}^{-2}$ in the spiral arms and $70 \mathrm{M}_{\odot} \mathrm{pc}^{-2}$ in the interarm region, has an implication on the gas distribution in the central few hundred parsecs. The average central gas surface density derived from the $\mathrm{CO}(2-1)$ luminosity is $260 \mathrm{M}_{\odot} \mathrm{pc}^{-2}$ over the same region. Our estimate being lower suggests that the gas may not be uniformly distributed. In fact, if the gas surface density were as high as the CO data imply, the flat gaseous disk would extinct $98 \%$ of the J-band light from the bulge behind it, and we would not see a moderation of that light caused by obscuration by the nuclear spiral, as we see in Fig. 1. Thus the gas density in the nuclear disk is most likely not as high, as the $\mathrm{CO}$ data imply. On the other hand, if we take the interarm gas surface density as the average density in the nuclear disk, only $27 \%$ of mass detected in $\mathrm{CO}$ may be distributed in the disk. Since within the CO beam size of $4.1^{\prime \prime} \times 3.1^{\prime \prime}$, a mass of $1.9 \times 10^{7} \mathrm{M}_{\odot}$ is implied by the $\mathrm{CO}$ emission, only as little as $0.5 \times 10^{7} \mathrm{M}_{\odot}$ would reside in the disk. The rest, $1.4 \times 10^{7} \mathrm{M}_{\odot}$ would be concentrated in the central few tens of parsecs. Hicks et al. (2009) estimated the gas fraction within a radius of $1.8^{\prime \prime}$ to be $f_{\text {gas }}=1.3 \pm 0.2 \%$. If most of the gas within the central $\mathrm{CO}$ beam were to come from an unresolved nuclear region, then the gas fraction there could be as much as $f_{\text {gas }} \sim 15 \%$, while being as little as $f_{\text {gas }} \sim 0.3 \%$ in the surrounding nuclear disk. Only observations at significantly higher resolution than those of Kohno et al. (2003) and Hsieh et al. (2008) would be able to confirm this conclusion. However, the low CO intensity in the regions between their central beams and the circumnuclear ring at a radius of $10^{\prime \prime}$ do suggest that such a low gas fraction is possible on scales of several hundred parsecs. It is possible that the explanation for the discrepancy between gas mass estimated from extinction and from $\mathrm{CO}$ emission may be even simpler. A conversion factor between the $\mathrm{CO}$ emission and the gas column density has to be assumed in order to obtain the gas masses and surface densities. As pointed out by Tacconi et al. (2008), around nuclei of galaxies this factor can be up to a few times smaller than its canonical value. If so, this would put the CO mass estimates in accordance with our estimates from extinction, and no gas concentration in an unresolved nuclear region would be required.

\subsection{Inflow Timescales and Nuclear Starbursts}

In Section 4.1 we showed that the width of the arms did not appear to change with radius, and so they could only be traced in to a radius of about $0.2^{\prime \prime}$, the point at which the gas dispersion begins to increase. This angular distance corresponds to $15-20 \mathrm{pc}$, strongly suggesting that the inflow along the arms is not feeding the AGN directly. It is on these scales that a nuclear starburst has been found, both by StorchiBergmann et al. (2005) and also by Davies et al. (2007) who showed in their Fig. 20 that the Br $\gamma$ flux did appear marginally resolved, covering the central $0.4-0.5^{\prime \prime}$. We conclude therefore that the arms are feeding 
a gas reservoir in the central few tens of parsecs, and hence giving rise to starbursts on these scales.

In NGC 1097, the data presented by Storchi-Bergmann et al. (2005) and Davies et al. (2007) are consistent with a recent starburst creating $10^{6} \mathrm{M}_{\odot}$ of stars in a very short burst that occurred from 1 to $10 \mathrm{Myr}$ previously. For the inflow rate of $0.06 \mathrm{M}_{\odot} \mathrm{yr}^{-1}$ that we estimated in Section 6, it would take $16 \mathrm{Myr}$ to provide sufficient gas for such a starburst, assuming $100 \%$ star forming efficiency. For a more realistic efficiency closer to $10 \%$, the timescale would increase to order $150 \mathrm{Myr}$. Thus, for a steady-state inflow, one could expect there to be episodic starbursts, recurring on timescales of order 20-150 Myr. For such a scenario, we would currently be in the phase shortly after a burst.

If the inflow rate estimated in this paper remains unchanged, gas in the nuclear disk within the central CO beam, whose mass we estimated at $0.5 \times 10^{7} \mathrm{M}_{\odot}$, is sufficient to last for just five starburst events. However, Hsieh et al. (2008) estimate the total gas mass inwards from the nuclear ring, i.e. at radii below $8^{\prime \prime}$ at $2.4 \times 10^{8} \mathrm{M}_{\odot}$, which, after correcting for the conversion factor between the $\mathrm{CO}$ emission and the gas column density adopted in this paper gives $1.1 \times 10^{8} \mathrm{M}_{\odot}$. This implies an average gas surface density of $75 \mathrm{M}_{\odot} \mathrm{pc}^{-2}$, virtually the same as our estimate for the nuclear disk within the central CO beam. Steadystate inflow in the nuclear spiral of $0.06 \mathrm{M}_{\odot} \mathrm{yr}^{-1}$ needs $1.8 \mathrm{Gyr}$ to drain this reservoir. It is also possible that this reservoir may be refilled from the more massive nuclear ring on a similar rate. Thus the gas dynamics inside the nuclear ring implied by the observed morphology and kinematics, may represent a state that can be sustained for long timescales. The nuclear spiral is sufficient to play the role of a mechanism that feeds gas to the innermost parsecs of a galaxy, where recurrent star-forming activity takes place. Inflow in the nuclear spiral is up to two orders of magnitude smaller than in the bar, but it is consistent with the observed properties of the nuclear stellar population, and represents a mode of flow that is sustainable for about a Gigayear.

\subsection{Torus}

If the unresolved nuclear region contains a gas reservoir that supplies recurrent starbursts, an important question concerns how this gas reservoir relates to the canonical molecular obscuring torus. This topic has been addressed in detail by Davies et al. (2006) for NGC 3227, Mueller Sanchez et al. (2009) for NGC 1068, and by Hicks et al. (2009) for a sample of 9 Seyfert galaxies. Their conclusion is that the torus is made up of a number of sub-components that fulfil different roles; and that the molecular gas concentrations on scales of tens of parsecs are associated with the outer extent of the overall structure. This picture is consistent with mid-infrared interferometric data that have revealed both a bright compact and a more clumpy extended structures in Circinus (Tristram et al. 2007) and NGC 1068 (Raban et al. 2009); and also with the simulations performed by Schartmann et al. (2009) (their Fig. 2) which suggest there may be a compact turbulent disk surrounded by a larger scale component comprising dense inflowing gas filaments embedded in a hot medium. Crucially, the structure in the simulations arose as a result of stellar evolution on scales of tens of parsecs.

In order to try and understand the physical state of the ISM, and how it could be related to the recurrent starburst episodes, Vollmer et al. (2008) developed a scenario linking these different evolutionary phases together. In that paper, the authors argued that a short starburst occurs during an episode of massive gas inflow to the central few tens of parsecs. And that during the subsequent supernovae phase, the diffuse intercloud medium is removed, leaving behind an ISM dominated by compact dense clumps. The star formation efficiency in this collisional disk is very low. The energy to support the scale height is instead supplied by gas accretion from larger scales; and is redistributed and dissipated via predominantly elastic 
cloud collisions, which are in principle possible if the magnetic fields are sufficiently strong. As such, the paper addresses the issue of what might be supporting the large scale height of the disk in the central tens of parsecs. Hicks et al. (2009) show that this appears to be a common phenomenon in Seyfert nuclei and discuss a number of possibilities that might explain it. They conclude that the AGN itself cannot contribute at the radii in question; that stellar outflows and supernovae also cannot contribute significantly; and that stellar radiation pressure could do so, although only during the active star forming phase. The alternative of converting gravitational energy of inflowing gas into turbulence, as suggested by Vollmer et al. (2008), did seem a possible mechanism as long as cloud collisions were sufficiently elastic. For the 6 galaxies analysed by these authors, their model predicted high inflow rates of several solar masses per year for 2 (including NGC 1068), and lower inflow rates of less than $1 \mathrm{M}_{\odot} \mathrm{yr}^{-1}$ for the other 4 (among which was NGC 1097). Notably, a high inflow rate for NGC 1068 was also reported by Mueller Sanchez et al. (2009) from a detailed analysis of the gas kinematics and distribution in the central $30 \mathrm{pc}$ of that galaxy. For NGC 1097, the results presented here tend to support the low $0.6 \mathrm{M}_{\odot} \mathrm{yr}^{-1}$ accretion rate predicted. However, when comparing the two values, one should bear in mind that the inflow rate estimated here is from scales of several hundred parsecs down to a few tens of parsecs, while that predicted by Vollmer et al. (2008) is from a few tens of parsecs down to a few parsecs. In addition, we note that in the model of Vollmer et al. (2008), the mass inflow rate depends on the gas mass and its dispersion as $\dot{M} \propto \sigma^{2} M_{\text {gas }}^{2}$. Thus, if the gas mass is overestimated by a factor of 2 , the required mass accretion rate is reduced by a factor 4 . In this way, moderate uncertainties on these properties can lead to a large uncertainty on the inflow rate. Despite the differences and uncertainties, both results point to the same conclusion that the gas inflow rate in the central regions of NGC 1097 is rather low.

\subsection{Origin of the spiral shock}

The morphology and amplitude of the observed kinematic spiral, presented in section 4.3 and analyzed in Section 5, are fully consistent with the nuclear spiral in NGC 1097 being a density wave in the gaseous disk, associated with a shock, and driven by a rotating gravitational potential. The driver can be identified with one of the two bars previously reported in NGC 1097 (see Prieto et al. 2005 for references), or with a possible companion or an orbiting mass. Nuclear spirals are driven inside the Inner Lindblad Resonance (ILR) of the driver, if one exists. The analysis of the rotation curve in NGC 1097 by Storchi-Bergmann et al. (1996) indicates that the outer bar definitely has an ILR, hence it can generate a nuclear spiral. It is possible that the inner bar in NGC 1097 also has an ILR for the following reason. The rotation curve in NGC 1097 reaches a plateau at very small radii: about 4- $5^{\prime \prime}$, as implied from $\mathrm{CO}$ and N[II] observations by Hsieh et al. (2008) and Fathi et al. (2006), and as small as 1.5" implied by Hicks et al. (2009) from $\mathrm{H}_{2}$ data. For a flat rotation curve, the ILR appears at 30\% of the corotation radius. The inner bar in NGC 1097 ends within the nuclear ring, hence it does not extend further than $8^{\prime \prime}$. Numerical models indicate that the corotation of an inner, nested bar, is located 2-3 times beyond its end (see Maciejewski \& Athanassoula 2008, Table 2). Thus the ILR of the inner bar in NGC 1097 should occur at 5-7", which remains in accordance with the rotation curve being flat in that region. It is possible therefore that the inner bar generates its own density wave within the radius of $5-7^{\prime \prime}$.

Maciejewski (2004b) constructed hydrodynamical models that enabled him to study in detail nuclear spirals associated with a shock in gas and generated by a large-scale bar. In all models 2-arm spirals are generated. It should be expected, given that the bar is an $m=2$ perturbation in the potential. However, in NGC 1097, we observe a 3-arm morphological spiral. Since another, inner bar is likely present in NGC 1097, 
and, as argued above, it can generate its own spiral pattern, possible non-linear interaction between the two nested bars in NGC 1097 may be responsible for the occurrence of a possibly transient third arm. This effect however has never been demonstrated in models. To the contrary, recent hydrodynamical models by Namekata et al. (2009) of gas flows in nested bars report shocks in the secondary inner bar, but these shocks are still associated with 2-arm spirals. Namekata et al. (2009) identified one of their models with the dynamics observed in the centre of NGC 1097, but, aside for the reason above, that model shows a different mode of gas flow, because a leading spiral, absent in NGC 1097, is present there between the nuclear ring and the central region.

A 3-arm steady-state spiral in the central gaseous disc of a galaxy was observed only in one type of model, when it was driven by an orbiting point mass (Etherington \& Maciejewski 2006). These models were constructed in order to study possible signatures of remnant black holes that orbit centres of galaxies. Etherington \& Maciejewski (2006) found that a $10^{7} \mathrm{M}_{\odot}$ black hole orbiting at 1-kpc radius generates a 3-arm spiral in the inner few hundred parsecs of a gaseous disc, but this spiral is weak and tightly wound, unlike the spiral shock that we observe in NGC 1097. However, no dependence on gas velocity dispersion was studied, which in NGC 1097 is higher than in the models.

Intriguingly for this scenario, Higdon \& Wallin (2003) showed that the X-shaped jet-like filaments extending from the nuclear region of NGC 1097 to $50 \mathrm{kpc}$ scales could be explained if NGC 1097 had captured a smaller companion in its central kiloparsecs of order $2 \mathrm{Gyr}$ previously. However, it seems unlikely that a small companion could host a black hole as massive as that required by the models to generate the 3 -arm spiral.

There are claims that the inflow in NGC 1097 can be of magnetic origin (Beck et al. 2005), but they do not attempt to reproduce the observed gas morphology. Another claim sometimes made about the origin of kinematic spirals in centres of galaxies in general, is that because of the obscuration one penetrates to different heights above the disk in the arm and between the arms, and because rotation velocity decreases with that height, one sees different velocities in the arm and between the arms, even if in the disk only axially symmetric rotation is present. However, if this was the origin of the kinematic spiral, it would trace the path of the morphological spiral, would have the same number of arms, and the residual velocities would be zero on the kinematic minor axis. None of these three characteristics is true about the kinematic spiral observed in NGC 1097, hence the explanation from obscuration has to be rejected. Interestingly, this also applies to the kinematic spiral observed by Fathi et al. (2006) in the [NII] emission line, which therefore also cannot be an effect of the (much greater optical) obscuration.

Thus, given our observations of NGC 1097, we face an unsettling state of affairs. The morphology and kinematics of the observed nuclear spiral have all properties of the spiral shock (velocity residuals, location of warm molecular gas upstream from the spiral in extinction, $m-1$ multiplicity of the kinematic spiral, relation of pitch angles of photometric and kinematic spiral), but we do not know what generates this shock. The most promising solution is the non-linear interaction of two density waves generated by the two bars, but more exotic hypotheses, like orbiting dark matter, cannot be excluded.

\section{Conclusions}

We have presented near infrared integral field spectroscopic observations of the central few arcsec (a few hundred parsecs) of NGC 1097 at a spatial resolution of $0.2^{\prime \prime}(20 \mathrm{pc})$, focusing on the distribution and kinematics of the stars and molecular gas. Our main conclusions are: 
- We confirm the 3-arm spiral pattern seen previously, and show that this must be a passive effect on the stellar continuum since there is no evidence for non-circular motions in the stellar kinematics.

- We show that the arms are also traced by $\mathrm{H}_{2}$, and that the shocked gas lies on the inside edge of the arm. After subtracting an axisymmetric disk model, the residual velocity field shows a clear 2arm spiral. Such characteristics are expected if the observed nuclear spiral is a density wave in gas associated with a shock.

- We use the contrast in stellar intensity between the inter-arm and arm regions, as well as the spectral slope of the stellar continuum, to estimate the extinction through the galaxy disk. From this we derive the inter-arm gas mass surface density to be $\sim 70 \mathrm{M}_{\odot} \mathrm{pc}^{-2}$, and that of the arms to be a factor 2 greater.

- A simple estimate of the gas inflow rate along the arms yields $\sim 1.2 \mathrm{M}_{\odot} \mathrm{yr}^{-1}$. However, in a spiral density wave there is outflow between the arms that partially cancels inflow in the arms, hence the net inflow rate is smaller than the simple estimate above. Numerical models indicate that the inflow rate could be 20 times smaller, i.e. about $0.06 \mathrm{M}_{\odot} \mathrm{yr}^{-1}$ in $\mathrm{NGC} 1097$. This is sufficient to generate recurrent starbursts, of a scale comparable to that observed, every 20-150 Myr.

- The nuclear spiral plays the role of a mechanism that feeds gas to the innermost parsecs of a galaxy, where recurrent episodes of star formation take place. The inflow rate, although vastly less than that induced by the large scale bar to the $800 \mathrm{pc}$ circumnuclear ring, is consistent with the observed properties of the nuclear stellar population, and represents a mode of inflow that is sustainable over timescales of $1 \mathrm{Gyr}$.

The authors are grateful to all those at MPE and at Paranal Observatory who were involved in obtaining these data; to Almudena Prieto for sharing her NACO imaging data; to Kambiz Fathi for sharing his [NII] residual velocity data; and to Bernd Vollmer for interesting and useful discussions. WM acknowledges an Academic Fellowship from Research Councils UK. Finally, the authors thank the referee for carefully reading the manuscript and making a number of comments that have helped to improve it.

Facilities: VLT (SINFONI)

\section{A. Linear approximation for density and l.o.s. velocity distribution in spiral density waves driven by a rotating potential}

Density waves in a gaseous disc can be described by analytic solution in the linear approximation under assumption of tightly wound spiral arms (for a derivation see Section 6.2.2 of Binney \& Tremaine 1987, hereafter BT622). Usually the solution is given for self-amplifying waves in self-gravitating gas, but a similar solution holds for waves driven in a gaseous disc by an external rotating gravitational potential (e.g. a gaseous disc inside a stellar bar). This solution has been thoroughly studied (Goldreich \& Tremaine 1979; Maciejewski 2004a), and here we emphasize its features relevant to the interpretation of the data presented in this paper. We follow the notation from Maciejewski (2004a), who in turn derived the solution for driven waves by adopting the derivation for self-amplifying waves given in BT622.

The shape of any arbitrary m-arm spiral can be described in polar coordinates $(R, \phi)$ by

$$
m \phi=f(R) .
$$


The pitch angle $\alpha$ of this spiral is given by

$$
\tan \alpha=\frac{\mathrm{d} R}{R \mathrm{~d} \phi}=\frac{m}{R \mathrm{~d} f / \mathrm{d} R}=\frac{m}{k R},
$$

where the last equality can be written under assumption that the spiral arm is tightly wound, and then $k \equiv d f / d R$ is the radial wavenumber.

The solution for the driven density wave is obtained from the linearized equations of gas dynamics. It gives the dispersion relation, i.e. the solution for $k$, which for the driven waves is the same as in the case of self-amplifying waves (BT622, Eq. 6-40; see also Eq. 16 in Maciejewski 2004a). After neglecting self-gravity in gas it takes the form

$$
k^{2}(R) c^{2}=m^{2}\left(\Omega(R)-\Omega_{p}\right)^{2}-\kappa^{2}(R),
$$

where $c$ is the sound speed in gas, $\Omega_{p}$ is the pattern speed of the driver, $\Omega(R)$ is the angular velocity in the gaseous disc, and $\kappa^{2} \equiv 2 \Omega(R d \Omega / d R+2 \Omega)$ is the epicyclic frequency squared. Having $k$, one can recover the shape of the spiral from Eq. (A2). After reshuffling Eq. (A3), the wave number can be written as

$$
k=\left(1-\frac{\Omega_{p}}{\Omega}\right) \eta \frac{m \Omega}{c},
$$

where $\eta=\sqrt{1-\frac{\kappa^{2}}{m^{2}\left(\Omega-\Omega_{p}\right)^{2}}}$. Well inside the corotation of the driver, $\eta$ takes values slightly less than one for $m \geq 3$. This $k$ substituted to Eq. (A2) gives

$$
\tan \alpha=\frac{c}{V_{\text {rot }}} \eta^{-1}\left(1-\Omega_{p} / \Omega\right)^{-1}
$$

Thus well inside the corotation of the driver, the pitch angle of the spiral $\alpha$ is proportional to the ratio of sound speed in gas $c$ to the rotation velocity $V_{\text {rot }}$. This ratio should be small for the linearization in the tightly wound limit to be applicable.

The linear solution for driven density waves gives also the distribution of each hydrodynamical variable $X$ (e.g. density $\rho$, velocity) in the disc plane. In the linear approximation, these variables are written as the sum of a zeroth-order axisymmetric term $X_{0}$, and first-order perturbation $X_{1}$, which in turn can be expanded in polar coordinates $(R, \phi)$ as a sum of terms with various multiplicities $m$, rotating at various rates $\Omega_{p}$ :

$$
X_{1}(R, \phi, t)=\operatorname{Re} \sum_{m, \Omega_{p}} X_{a}(R) e^{i m\left(\phi-\Omega_{p} t\right)} .
$$

For driven waves, only terms in Eq. (A6) with $\Omega_{p}$ equal to the pattern speed of the driver are excited. For a given gravitational potential $\Phi_{a}(R)$ and density distribution $\rho_{a}(R)$, radial and tangential velocity components, $v_{R a}$ and $v_{\phi a}$ are given by Eqs. (10) in Maciejewski (2004a). Because these equations contain terms with derivatives, and in $H_{a}=\Phi_{a}+c^{2} \rho_{a} / \rho_{0}$, gas density $\rho_{a}$ rapidly oscillates with radius $R$, terms with $\rho_{a}$ in derivative will dominate. Since for a tightly wound spiral $\mathrm{d} X / \mathrm{d} R \approx i k R$, equations for velocity components take the form

$$
v_{R a}=-\frac{m\left(\Omega-\Omega_{p}\right)}{k} \frac{\rho_{a}}{\rho_{0}}, \quad \quad v_{\phi a}=-\frac{i \kappa^{2}}{2 k \Omega} \frac{\rho_{a}}{\rho_{0}},
$$

which is the same as that for a self-amplifying wave (BT622, Eq. 6-37), after neglecting there $\Phi_{a}$, because the gravitational potential does not vary rapidly with radius for driven waves in gas with negligible self-gravity. 
The functional form of $\rho_{a}$ for a spiral wave that has density maxima at points defined by Eq. (A1) is

$$
\rho_{a}(R)=g(R) e^{-i f(R)}=\epsilon \rho_{0} e^{-i f(R)}
$$

where we assumed that the amplitude of the density perturbation $g(R)$ is proportional to the the unperturbed density $\rho_{0}$ with $\epsilon$ proportionality constant. Substituting Eq. (A8) and Eq. (A7) to Eq. (A6) gives first-order perturbation of gas density and velocity field

$$
\begin{gathered}
\rho_{1}(R, \phi, t)=\operatorname{Re}\left\{\epsilon \rho_{0} e^{-i f(R)+i m\left(\phi-\Omega_{p} t\right)}\right\}=\epsilon \rho_{0} \cos \left(m \phi-f(R)-m \Omega_{p} t\right) \\
v_{R 1}(R, \phi, t)=\operatorname{Re}\left\{-\frac{m\left(\Omega-\Omega_{p}\right)}{k} \frac{\rho_{a}}{\rho_{0}} e^{i m\left(\phi-\Omega_{p} t\right)}\right\}=\operatorname{Re}\left\{-\frac{m\left(\Omega-\Omega_{p}\right)}{k} \epsilon e^{-i f(R)+i m\left(\phi-\Omega_{p} t\right)}\right\}= \\
-\epsilon \frac{m\left(\Omega-\Omega_{p}\right)}{k} \cos \left(m \phi-f(R)-m \Omega_{p} t\right) \\
v_{\phi 1}(R, \phi, t)=\operatorname{Re}\left\{-\frac{i \kappa^{2}}{2 k \Omega} \frac{\rho_{a}}{\rho_{0}} e^{i m\left(\phi-\Omega_{p} t\right)}\right\}=\operatorname{Re}\left\{-\frac{i \kappa^{2}}{2 k \Omega} \epsilon e^{-i f(R)+i m\left(\phi-\Omega_{p} t\right)}\right\}=\epsilon \frac{\kappa^{2}}{2 k \Omega} \sin \left(m \phi-f(R)-m \Omega_{p} t\right)
\end{gathered}
$$

The term $m \Omega_{p} t$ indicates rigid rotation with pattern speed of the driver. We drop it in the remaining part of this Appendix, which corresponds to presenting the spiral at $t=0$. Substituting $k$ from Eq. (A4) to Eq. (A10) allows one to write the radial component of gas velocity as

$$
v_{R 1}=-\frac{\epsilon c}{\eta} \cos (m \phi-f(R))
$$

Thus in the linear approximation, if the arm/interarm density ratio in the spiral is $\frac{1+\epsilon}{1-\epsilon}$, the amplitude of the radial flow is $\epsilon c$, where $c$ is the speed of sound in gas. This shows maximal inflow/outflow attainable in the linear flow.

The complete velocity components are the sums of the unperturbed zeroth-order term, which for the tangential velocity component is the rotation velocity $V_{\text {rot }}$, and the first order perturbation:

$$
v_{R}=0+v_{R 1}=v_{R 1} \quad v_{\phi}=V_{r o t}+v_{\phi 1}
$$

The observed line-of-sight velocity is $v_{l o s}=v_{y} \sin i$, where $i$ is the inclination of the disc and

$$
v_{y}=v_{R} \sin \phi+v_{\phi} \cos \phi=V_{\text {rot }} \cos \phi-\epsilon\left[\frac{m\left(\Omega-\Omega_{p}\right)}{k} \cos (m \phi-f(R)) \sin \phi-\frac{\kappa^{2}}{2 k \Omega} \sin (m \phi-f(R)) \cos \phi\right]
$$

The same result was obtained by Canzian \& Allen (1997), who studied density perturbation in spiral coordinates. Inside the corotation of the driver, the coefficient at the first component of the sum in brackets is positive, while the one at the second component is negative. If both coefficients were of the same magnitude, they would sum up to a single component with angle dependence $\sin ((m-1) \phi-f(R))$. This is the original finding by Canzian (1993) that an $m$-arm density perturbation appears as an $m$-1-arm kinematic wave. More accurately, $a \cos \alpha \sin \beta-b \sin \alpha \cos \beta=\frac{a-b}{2} \sin (\alpha+\beta)-\frac{a+b}{2} \sin (\alpha-\beta)$, which applied to Eq. (A14) gives

$$
v_{y}=V_{r o t} \cos \phi+\frac{\epsilon}{2 k}\left[\left(m\left[\Omega-\Omega_{p}\right]+\kappa^{2} / 2 \Omega\right) \sin ([m-1] \phi-f(R))-\left(m\left[\Omega-\Omega_{p}\right]-\kappa^{2} / 2 \Omega\right) \sin ([m+1] \phi-f(R))\right]
$$

Thus the $m$-arm photometric spiral, results in a combination of kinematic spirals with $m-1$ and $m+1$ arms, whose amplitude ratio is $\frac{m\left(1-\Omega_{p} / \Omega\right)+\kappa^{2} / 2 \Omega^{2}}{m\left(1-\Omega_{p} / \Omega\right)-\kappa^{2} / 2 \Omega^{2}}$. Well within the corotation of the driver this ratio takes values 
between $\frac{m+2}{m-2}$ and $\frac{m+\frac{1}{2}}{m-\frac{1}{2}}$, hence the spiral of $m-1$ arms dominates. The $m-1$ component from Eq. (A15) corresponds to the spiral shape defined by $(m-1) \phi=f(R)$. Because $f(R)$ is the same for the photometric and the kinematic spiral, Eq. (A2) implies that

$$
\frac{\tan \alpha_{k i n}}{\tan \alpha_{\text {phot }}}=\frac{m-1}{m}
$$

and the kinematic spiral is more tightly wound, with the ratio of pitch angles constant inside the corotation of the driver.

In Fig. 8, we visualize the appearance of a 3-arm photometric spiral, and its corresponding kinematic spiral for a particularly simple case of a logarithmic spiral, which forms in a disk rotating with a constant

velocity. In this setup, in the region well inside the corotation of the driver $\left(\Omega \gg \Omega_{p}\right), \eta=\sqrt{1-\frac{2}{m^{2}}}$ is a constant, and the amplitude ratio of the $m-1$-arm and $m+1$-arm kinematic spirals is $\frac{m+1}{m-1}$. We show the distributions of the density, the radial velocity in the disc plane and the residual line-of-sight velocity in the innermost $200 \mathrm{pc}$ for parameters close to those of the nucleus of NGC 1097: $V_{\text {rot }}=100 \mathrm{~km} / \mathrm{s}, c=50 \mathrm{~km} / \mathrm{s}$ and we use $\epsilon=0.1$.

\section{REFERENCES}

Abuter R., Schreiber J., Eisenhauer F., Ott T., Horrobin M., Gillesen S., 2006, NewAR, 50, 398

Athanassoula E., 1992, MNRAS, 259, 345

Ballantyne D., 2008, ApJ, 685, 787

Beck R., Fletcher A., Shukurov A., Snodin A., Sokoloff D., Ehle M., Moss D., Shoutenkov V., 2005 A\&A, 444,739

Binney J., Tremaine S., 1987, Galactic Dynamics. Princeton Univ. Press, Princeton, Section 6.2.2 (BT622)

Bonnet H., et al., 2004, The ESO Messenger, 117, 17

Calzetti D., Kinney A., Storchi-Bergamnn T., 1994, ApJ, 429, 582

Canzian B., 1993, ApJ, 414, 487

Canzian B., Allen R., 1997, ApJ, 479, 723

Cid Fernandes R., Gu Q., Melnick J., Terlevich E., Terlevich R., Kunth D., Rodrigues Lacerda R., Johuet B., 2004, MNRAS, 355, 273

Combes F., 2003, in Active Galactic Nuclei: from Central Engine to Host Galaxy, eds. Collin S., Combes F., Shlosman I., ASP Conference Series, vol. 290, p. 411

Cresci G., et al., 2009, ApJ, submitted

Davies R., Sternberg A., Lehnert M., Tacconi-Garman L., 2005, ApJ, 633, 105

Davies R., et al., 2006, ApJ, 646, 754

Davies R., 2007a, MNRAS, 375, 1099 
Davies R., 2007b in The 2007 ESO Instrument Calibration Workshop, eds Kaufer A. Kerber F., ESO Astrophysics Symposia, (Springer Berlin Heidelberg), p. 249

Davies R., Mueller Sanchez D., Genzel R., Tacconi L., Hicks E., Friedrich S., Sternberg A., 2007, ApJ, 671, 1388

Eisenhauer F., et al., 2003, in Instrument Design and Performance for Optical/Infrared Ground-based Telescopes, eds. Masanori I., Moorwood A., Proc. SPIE, 4841, 1548

Emsellem E., Greusard D., Combes F., Friedli D., Leon S., Pécontal E., Wozniak H., 2001, A\&A, 368, 52

Englmaier P., Shlosman I., 2000, ApJ, 528, 677

Etherington J., Maciejewski W., 2006, MNRAS, 367, 1003

Fabian A., Barcons X., Almaini O., Iwasawa K., 1998, MNRAS, 297, L11

Fathi K., Storchi-Bergmann T., Riffel R., Winge C., Axon D., Robinson A., Apetti A., Marconi A., 2006, ApJ, 641, L25

Fathi K., et al., 2008, ApJ, 675, L17

Förster Schreiber N., et al., 2009, ApJ submitted

García-Burillo S., Combes F., Schinnerer E., Boone F., Hunt L., 2005, A\&A, 441, 1011

Gerin M., Nakai N., Combes F., 1988, A\&A, 203, 44

Goldreich P., Tremaine S., 1979, ApJ, 233, 857

Hicks E., Davies R., Malkan M., Genzel R., Tacconi L., Mueller Sanchez F., Sternberg A., 2009, ApJ, submitted

Higdon J., Wallin J., 2003, ApJ, 585, 281

Hsieh P.-Y., Matsushita S., Lim J., Kohno K., Sawada-Satoh S., 2008, ApJ, 683, 70

Ho L., Filippenko A., Sargent W., 1997, ApJ, 487, 591

Hummel E., van der Hulst J., Keel W., 1987, A\&A, 172, 32

Hunt L., Malkan M., 2004, ApJ, 616, 707

Iyomoto N., Makishima K., Fukazama Y., Tashiro M., Ishisaki Y., Nakai N., Taniguchi Y., 1996, PASJ, 48, 231

Kennicutt Jr., R., 1998, ApJ, 498, 541

Kohno K., Ishizuki S., Matsushita S., Vila-Vilaró B., Kawabe R., 2003, PASJ, 55, L1

Kormendy J., Kennicutt Jr. R., 2004, ARA\&A, 42, 603

Kotilainen J., Reunanen J., Laine S., Ryder S., 2000, A\&A, 353, 834

Laine S., Shlosman I., Knapen J., Peletier R., 2002, ApJ, 567, 97 
Levenson N., Weaver K., Heckman T., 2001, ApJ, 550, 230

Li C., Kauffmann G., Heckman T., Jing Y., White S., 2008a, MNRAS, 385, 1903

Li C., Kauffmann G., Heckman T., White S., Jing Y., 2008b, MNRAS, 385, 1915

Maciejewski W., Teuben P., Sparke L., Stone J., 2002, MNRAS, 329, 502

Maciejewski W., 2004a, MNRAS, 354, 883

Maciejewski W., 2004b, MNRAS, 354, 892

Maciejewski W., 2006, in Mapping the Galaxy and Nearby Galaxies, eds Wada K., Combes F., (Springer)

Maciejewski W., Athanassoula E., 2008, MNRAS, 389, 545

Martini P., Regan M., Mulchaey J., Pogge R., 2003, ApJ, 589, 774

Mueller Sanchez F., Davies R., Genzel R., Tacconi L., Eisenhauer F., Hicks E., Friedrich S., Sternberg A., 2009, ApJ, in press

Mulchaey J., Regan M., 1997, ApJ, 482, L135

Namekata D., Habe A., Matsui H., Saitoh T., 2009, ApJ, submitted

Piner B. G., Stone J., Teuben P., 1995 ApJ, 449, 508

Prieto A., Maciejewski W., Reunanen J., 2005, AJ, 130, 1472

Raban D., Jaffe W., Röttgering H., Meisenheimer K., Tristram K., 2009, MNRAS, in press

Regan M., Teuben P., 2003, ApJ, 582, 723

Schartmann M., Meisenheimer K., Klahr H., Camenzind M., Wolf S., Henning Th., 2009, MNRAS, in press

Schinnerer E., Böker T., Emsellem E., Lisenfeld U., 2006, ApJ, 649, 181

Schmitt H., 2001, AJ, 122, 2243

Shlosman I., Juhan F., Begelman M., 1989, Nature, 338, 45

Shlosman I., Begelman M., Frank J., 1990, Nature, 345, 679

Shlosman I., Peletier R., Knapen J., 2000, ApJ, 535, L83

Spekkens K., Selwood J., 2007, ApJ, 664, 204

Storchi-Bergmann T., Baldwin J., Wilson A., 1993, ApJ, 410, L11

Storchi-Bergmann T., Wilson A., Baldwin J., 1996, ApJ, 460, 252

Storchi-Bergmann T., et al., 2003, ApJ, 598, 956

Storchi-Bergamnn T., Nemmen R., Spinelli P., Eracleous M., Wilson A., Filippenko A., Livio M., 2005, ApJ, 624, L13 
Storchi-Bergamnn T., Dors Jr. O., Riffel R., Fathi K., Axon D., Robinson A., Marconi A., Östlin G., 2007, ApJ, 670, 959

Tacconi L., et al., 2008, ApJ, 680, 246

Thompson T., Quartaert E., Murray N., 2005, ApJ, 630, 167

Tristram K., et al. 2007, A\&A, 474, 837

Tokunaga, A., 2000, in Allen's Astrophysical Quantities, 4th edition, eds Cox A., (Springer)

Vollmer B., Beckert T., Davies R., 2008, A\&A, 491, 441

Wada K., 2004, in Carnegie Observatories Astrophysics Series, Vol. 1: Coevolution of Black Holes and Galaxies ed Ho L., (Cambridge: Cambridge Univ. Press), p.186

Wada K., Norman C., 2002, ApJ, 566, L21 

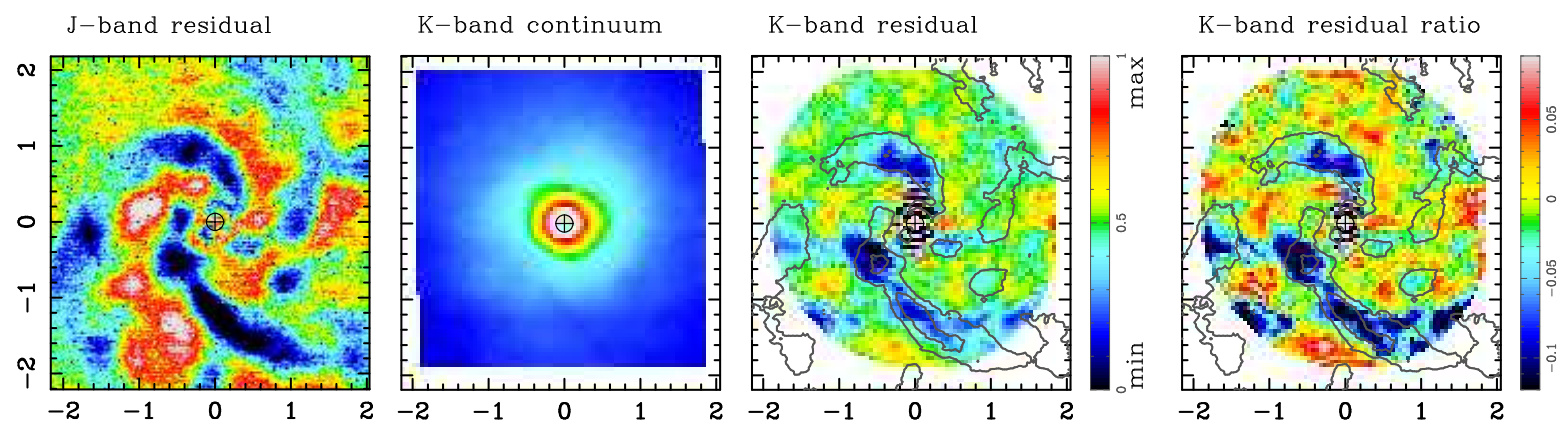

Fig. 1.- Far Left: NACO J-band continuum residual (from, and as presented in, Prieto et al. 2005) after subtracting elliptical isophotes. Middle Left: SINFONI K-band continuum, showing the bright nucleus containing a significant fraction of non-stellar light. Middle Right: the same K-band continuum after subtracting elliptical isophotes. Far Right: ratio of the residual to the smooth isophotal model (from which the central non-stellar point source has been subtracted). This allows one to read-off almost directly as $0.1-0.15$ the contrast ratio described in Section 4.2. Superimposed are contours outlining the negative J-band residual in the left-most panel. The same 3-armed spiral structure is apparent in both J-band NACO data and SINFONI K-band data. In all panels, north is up and east is left; and the scale in arcsec (with $1^{\prime \prime}=85 \mathrm{pc}$ ) is indicated.
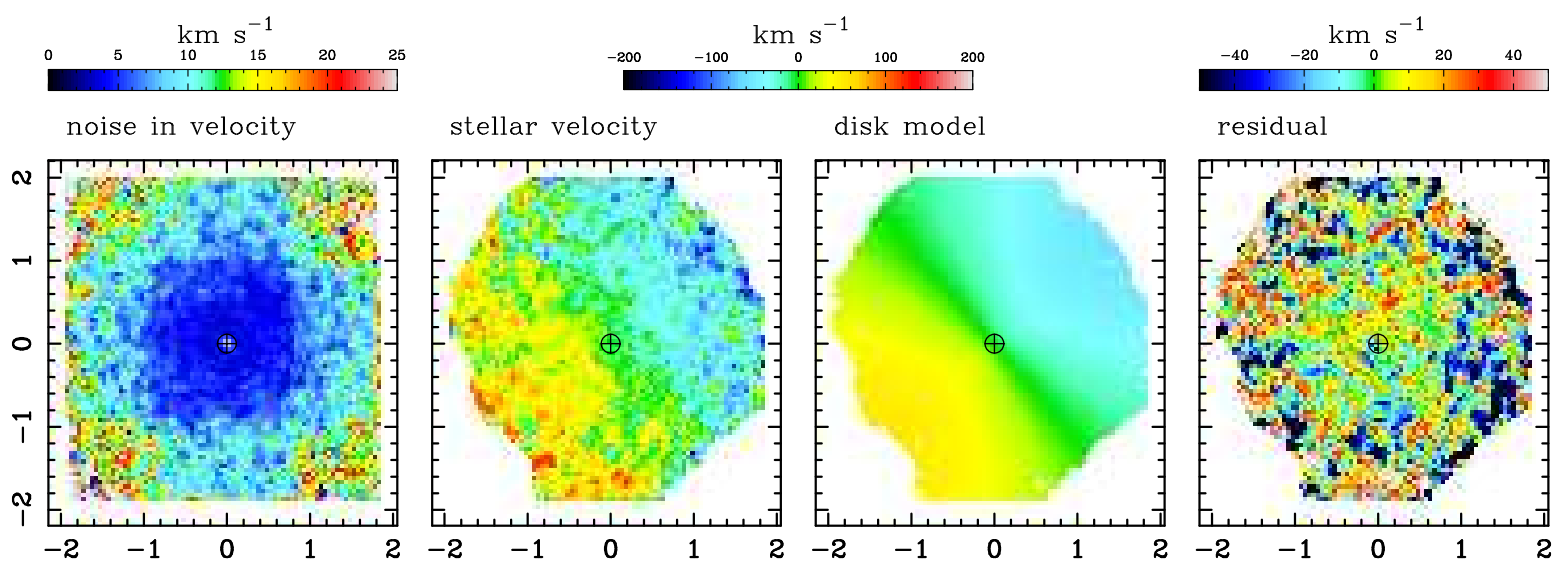

Fig. 2.- Far left: noise in stellar velocity field. The pattern is dominated by the effects of dithering (i.e. the central part of the field with the longest integration time has uncertainties of $5 \mathrm{~km} \mathrm{~s}^{-1}$ or less, and the corners have the least integration and highest errors). Center left: the stellar velocity field derived by fitting spectral templates to the $2.3 \mu \mathrm{m} \mathrm{CO}$ bandhead. Regions of particularly high noise have been excised. Center right: the best fitting axisymmetric disk model velocity field. Far right: the residual after subtracting the model velocity field from that observed shows no coherent structures above the noise level. In all panels, north is up and east is left; and the scale in $\operatorname{arcsec}$ (with $1^{\prime \prime}=85 \mathrm{pc}$ ) is indicated. 

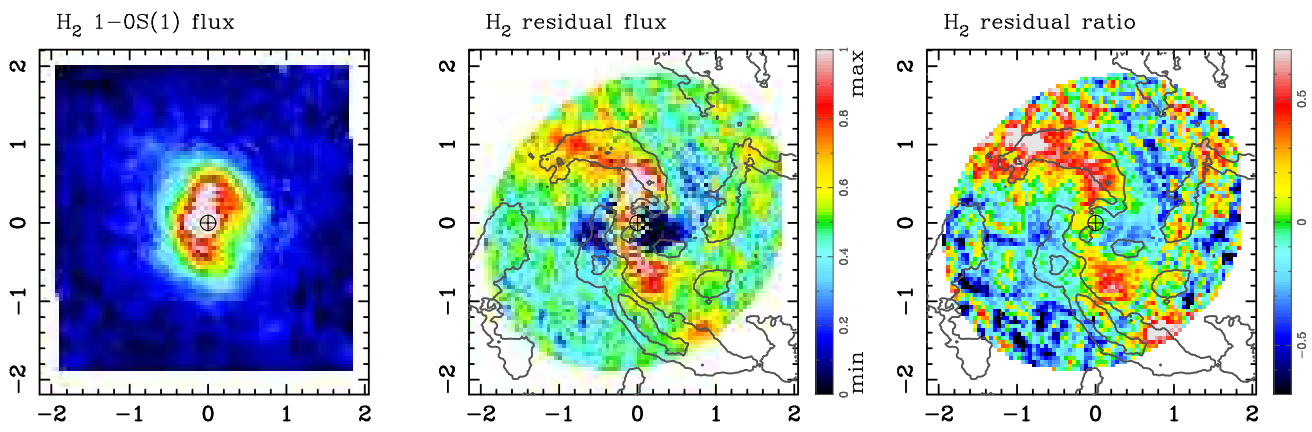

Fig. 3.- Left: $\mathrm{H}_{2}$ distribution as traced by the $2.12 \mu \mathrm{m} \mathrm{1-0} \mathrm{S(1)} \mathrm{line.} \mathrm{Middle:} \mathrm{the} \mathrm{flux} \mathrm{residual,} \mathrm{after}$ subtracting elliptical isophotes, reveals a 3 -armed spiral pattern (2 strong arms and 1 weak) similar to that seen in stellar absorption. In the flux map, the strength of the residual increases to within about $0.1^{\prime \prime}$ of the centre. Right: the ratio of the residual flux to the total flux at each spaxel shows a more uniform distribution along each arm. For reference, contours outlining the negative J-band stellar residual are superimposed. In all panels, north is up and east is left; and the scale in $\operatorname{arcsec}\left(\right.$ with $1^{\prime \prime}=85 \mathrm{pc}$ ) is indicated.

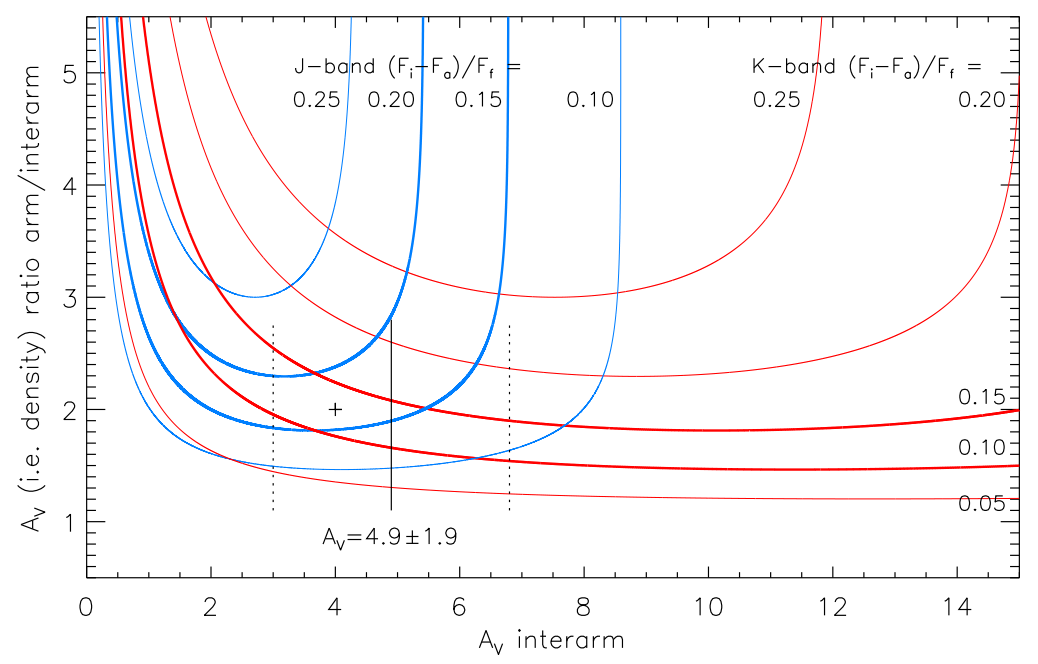

Fig. 4.- Plot of the arm-interarm extinction ratio as a function of interarm extinction, for several arminterarm contrast ratios (defined as $C=F_{i} /\left(F_{i}-F_{a}\right)$ ). Since $n_{H}\left(\mathrm{~cm}^{-} 2\right)=1.9 \times 10^{22} A_{V}$ (Tokunaga 2000), this is equivalently a plot of the arm-interarm density ratio. Lines for $C_{K}$ are drawn in red and $C_{J}$ in blue; the thick lines denote the boundaries for the respective measured values. The extinction in the central $2^{\prime \prime}$ measured from the spectral slope of the K-band continuum is also overplotted. Within the uncertainties, the constraints imply an interarm extinction of $A_{V}=4 \pm 1$ and an arm-interarm density ratio of $2.0 \pm 0.3$. The adopted value is shown as a plus sign. 

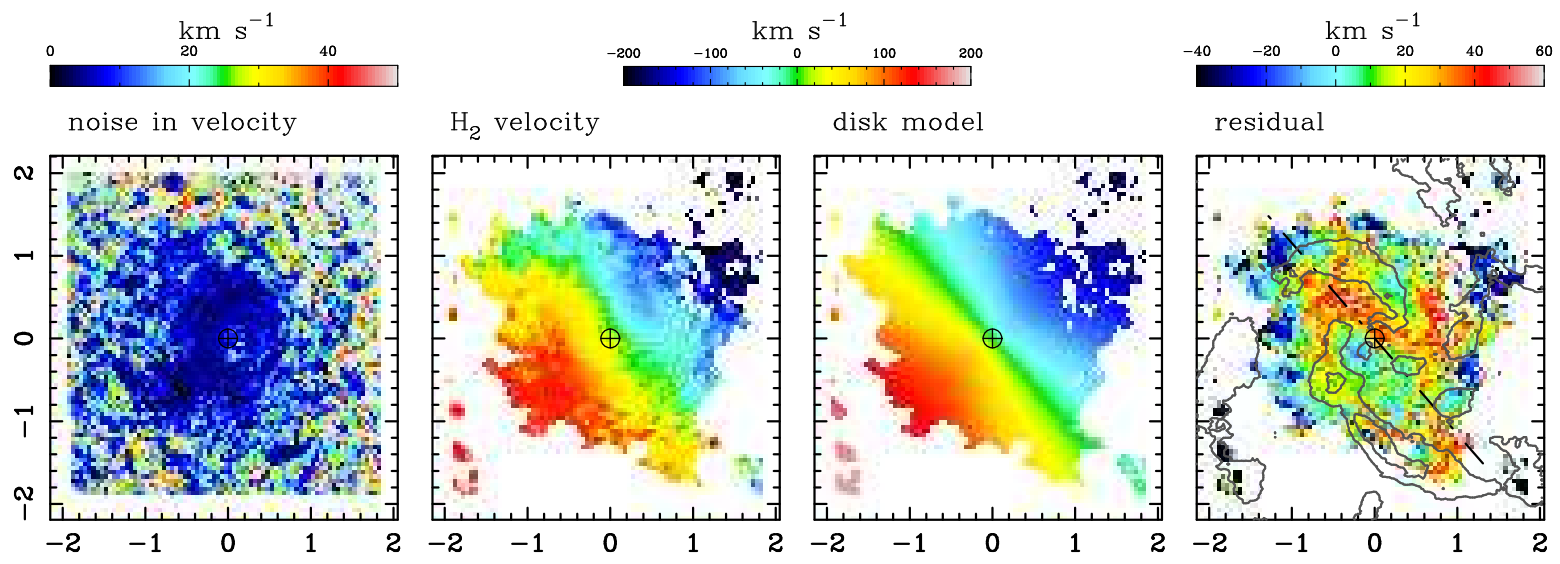

Fig. 5.- Far left: noise in $\mathrm{H}_{2}$ velocity field. The pattern is dominated by the effects of the flux distribution (i.e. the uncertainty is greatest in the outer regions where the flux is lowest). Center left: the $\mathrm{H}_{2}$ velocity field derived by fitting emission line templates to the $2.12 \mu \mathrm{m} 1-0 \mathrm{~S}(1)$ line. Regions of particularly high noise have been excised. Center right: the best fitting axisymmetric disk model velocity field. Far right: the residual after subtracting the model velocity field from that observed shows a clear 2-arm spiral pattern. Intrinsic velocities and residuals are about $50 \%$ higher than shown here due to the correction for inclination. The dashed line in this panel traces the minor axis of the galaxy; and the contours outline the negative J-band stellar residual. In all panels, north is up and east is left; and the scale in arcsec (with $1^{\prime \prime}=85 \mathrm{pc}$ ) is indicated.

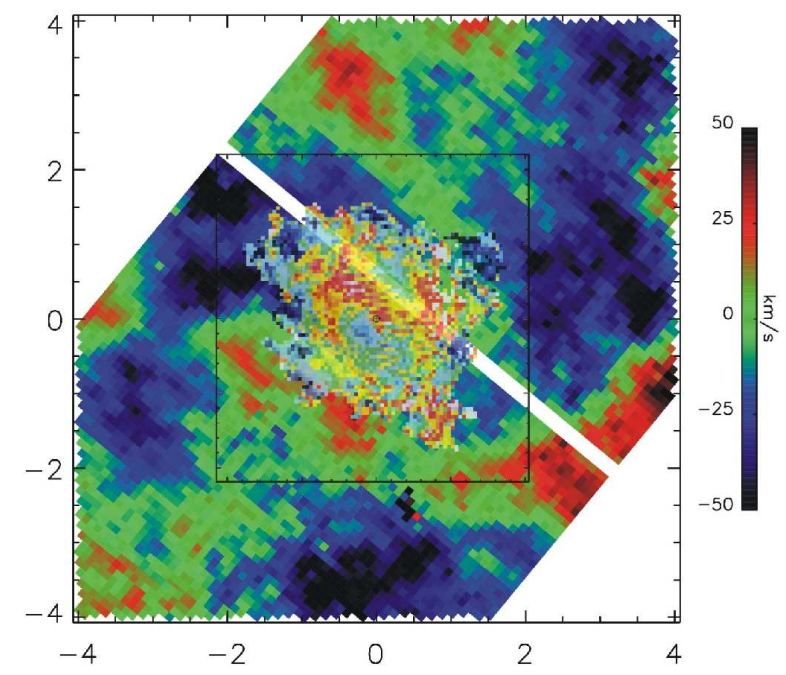

Fig. 6.- Velocity residuals of the [NII] ionised gas from Fathi et al. (2006). The SINFONI data are superimposed. Note that Fathi et al. (2006) identified 3 kinematic arms which they associated with the morphological arms. As we argue in the text, the kinematics appear to be consistent with those in the 1-0S(1) line, exhibiting a 2-arm signature - traced by positive velocity residual and separated by 2 regions of negative residual (as explained in the text, the only significant exception is immediately west of the nucleus). In this figure, north is up and east is left; and the scale in arcsec. 

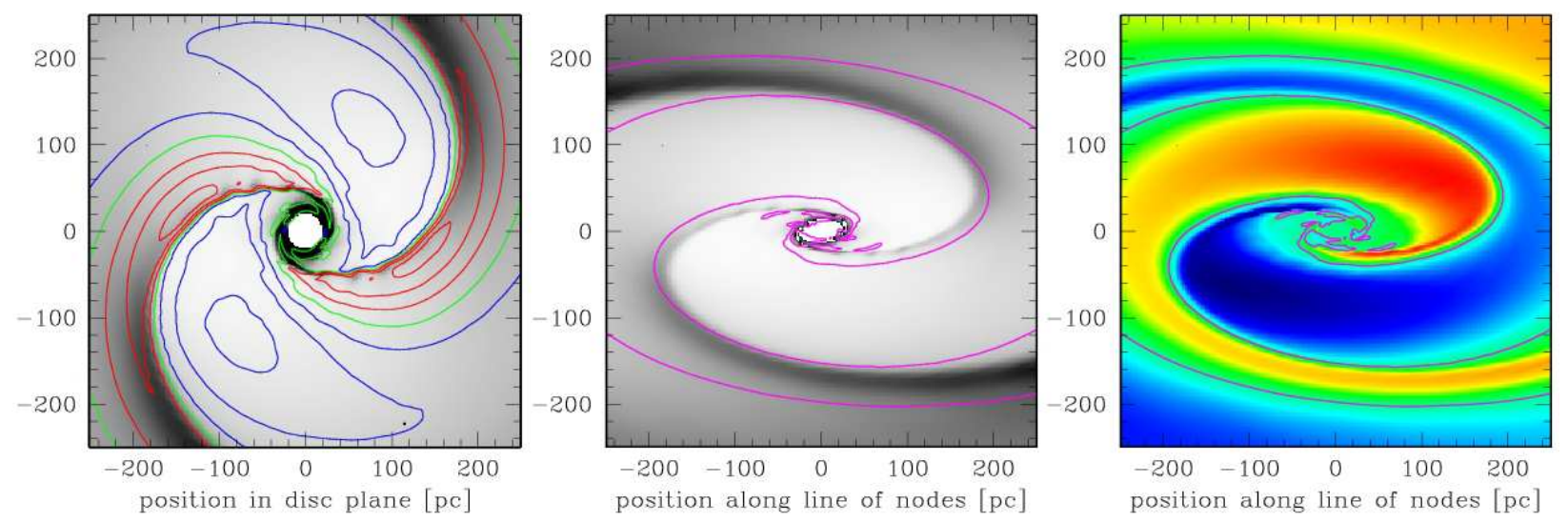

Fig. 7.- A snapshot of the nuclear spiral shock in gas in model 8S20r from Maciejewski (2004b) taken at $300 \mathrm{Myr}$, after the flow in the spiral stabilized. The gaseous disc is rotating clockwise. Left: Radial velocity (contours) plotted over gas density (grey-scale, darker shades mark higher density). Blue contours mark positive radial velocity (outflow), green contours mark zero radial velocity, and red contours mark negative radial velocity (inflow). The spacing between the contours is $20 \mathrm{~km} \mathrm{~s}^{-1}$. The central white hole is outside the inner (reflective) boundary of the polar grid in the model, hence the model provides no information about this region, and regions immediately adjacent to it may be influenced by boundary conditions. Centre: Photometric spiral in gas (grey-scale) as it would appear in the sky for the galaxy disc inclined at $60^{\circ}$. Pink contours mark zero line-of-sight velocity in gas. Right: Kinematic spiral in the residual gas velocity field. The colour scale ranges from $-55 \mathrm{~km} \mathrm{~s}^{-1}$ (blue) to $55 \mathrm{~km} \mathrm{~s}^{-1}$ (red). Pink contours are the same as in the middle panel. 


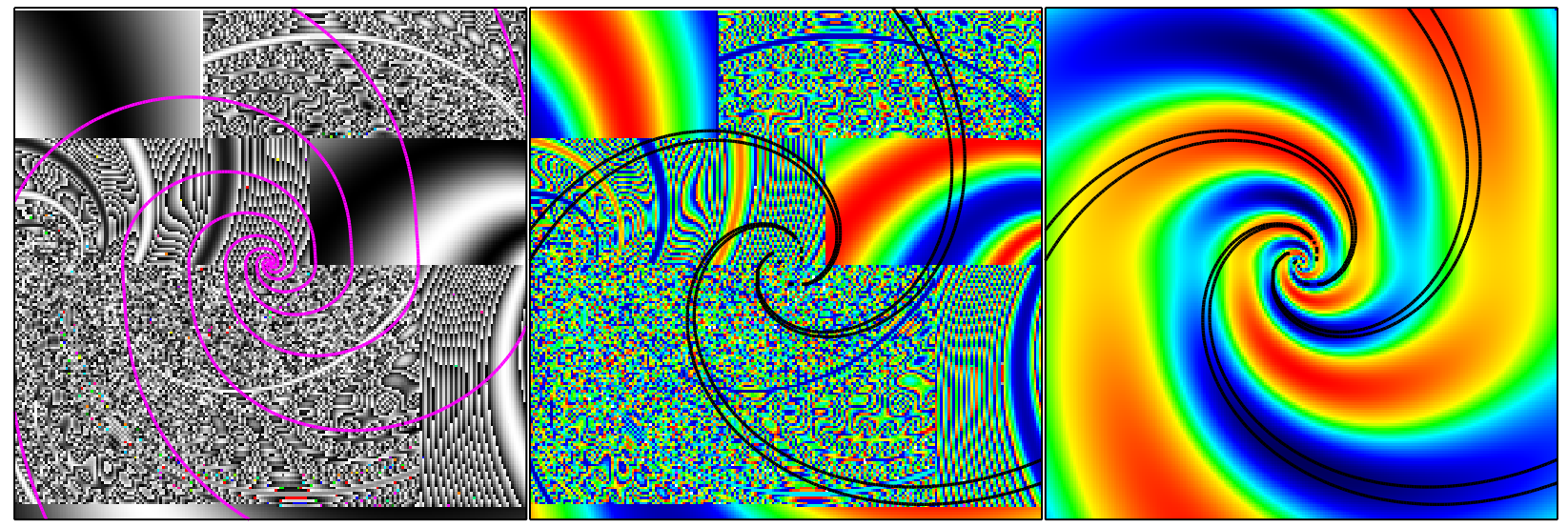

Fig. 8.- An example of a spiral density wave in gas in the linear approximation explored in Appendix A. The spiral is shown well inside of the corotation of the driver. Left: Morphological 3-arm spiral (grey-scale) with the residual LOS (i.e. observed) zero-velocity (pink contours). Centre: Radial velocity in the disc plane (colours: red for inflow, blue for outflow), with the density maxima in the spiral arms marked by black contours. This plot indicates that while morphological spiral arms are associated with inflow, there is outflow between the arms in spiral density waves. Right: The LOS residual velocity field (colours: red for positive, blue for negative) after subtraction of circular motions reveals a 2-arm kinematic spiral. The density maxima are marked by black contours as in the central panel. The line of nodes is horizontal, but the image is not projected, as this only involves scaling in the vertical direction. 